TRANSACTIONS OF THE

AMERICAN MATHEMATICAL SOCIETY

Volume 357, Number 10, Pages 4017-4042

S 0002-9947(05)03927-9

Article electronically published on May 25, 2005

\title{
INEQUALITIES FOR FINITE GROUP PERMUTATION MODULES
}

\author{
DANIEL GOLDSTEIN, ROBERT M. GURALNICK, AND I. M. ISAACS
}

\begin{abstract}
If $f$ is a nonzero complex-valued function defined on a finite abelian group $A$ and $\hat{f}$ is its Fourier transform, then $|\operatorname{supp}(f)||\operatorname{supp}(\hat{f})| \geq|A|$, where $\operatorname{supp}(f)$ and $\operatorname{supp}(\hat{f})$ are the supports of $f$ and $\hat{f}$. In this paper we generalize this known result in several directions. In particular, we prove an analogous inequality where the abelian group $A$ is replaced by a transitive right $G$-set, where $G$ is an arbitrary finite group. We obtain stronger inequalities when the $G$-set is primitive, and we determine the primitive groups for which equality holds. We also explore connections between inequalities of this type and a result of Chebotarëv on complex roots of unity, and we thereby obtain a new proof of Chebotarëv's theorem.
\end{abstract}

\section{INTRODUCTION}

The starting point for this paper is an inequality for complex-valued functions defined on finite abelian groups. This result is generally attributed to D. L. Donoho and P. B. Stark, although in their paper [4], they prove the result only for cyclic groups. (The more general statement, with a proof, can be found as Theorem 14.1 in the book by A. Terras [12.)

Theorem A (Donoho and Stark). Let $A$ be a finite abelian group and suppose that $f$ is an arbitrary nonzero complex-valued function on $A$. Writing $\hat{f}$ to denote the Fourier transform of $f$, we have

$$
|\operatorname{supp}(f)||\operatorname{supp}(\hat{f})| \geq|A| \text {. }
$$

Here, $\operatorname{supp}(f)$ is the support of $f$, which is the set of elements of $A$ on which the function $f$ takes nonzero values. Similarly, $\operatorname{supp}(\hat{f})$ is the set of linear characters of $A$ on which $\hat{f}$ takes nonzero values. In other words, a linear character $\lambda$ of $A$ lies in $\operatorname{supp}(\hat{f})$ precisely when $\lambda$ occurs with nonzero coefficient, when the function $f$ is written as a linear combination of the set $\hat{A}$ of linear characters of $A$.

We can think about Theorem A in the following much more general context. Let $S$ be a finite right $G$-set, where $G$ is an arbitrary group, and suppose that the action of $G$ on $S$ is transitive. (It will be no loss to assume that the action of $G$ on $S$ is faithful, and so we can assume that $G$ is finite.) Let $F$ be an arbitrary field and write $F[S]$ to denote the $F$-space of $F$-linear combinations of members of $S$.

Received by the editors October 24, 2003.

2000 Mathematics Subject Classification. Primary 20B05; Secondary 20B15, 42 A99.

The research of the second author was partially supported by Grant DMS 0140578 of the U.S. NSF.

The research of the third author was partially supported by the U.S. NSA.

(C)2005 American Mathematical Society Reverts to public domain 28 years from publication 4017 
(Alternatively, we could view this space as the set of $F$-valued functions on $S$, but we prefer the former point of view, in which $S$ is a subset of $F[S]$.) If we extend the given action of $G$ on $S$ linearly to all of $F[S]$, then $F[S]$ becomes a right $G$-module over $F$ : the permutation module. Now if $v \in F[S]$, we write $\operatorname{supp}(v)$ to denote the set of points of $S$ that occur with nonzero coefficients in $v$. (This, of course, is exactly the support of the function $f$ corresponding to the vector $v$, where $v$ and $f$ are related by the equation $v=\sum_{s \in S} f(s) s$.)

Returning now to the situation of Theorem $\mathrm{A}$, take $F=\mathbb{C}$, the complex numbers, and $G=S=A$, where the transitive action of $G$ on $S$ is the regular action, defined by multiplication in $A$. In the theorem, we are given a function $f$ defined on $A$, and we let $v \in F[S]=\mathbb{C}[A]$ be the corresponding vector, so that $\operatorname{supp}(f)=\operatorname{supp}(v)$. But how can we interpret $\operatorname{supp}(\hat{f})$ in terms of the vector $v$ ?

View $F[S]=\mathbb{C}[A]$ as the complex group algebra of $A$, and write $e_{\lambda} \in \mathbb{C}[A]$ to denote the idempotent corresponding to the linear character $\lambda$ of $A$. (Recall that $e_{\lambda}=(1 /|A|) \sum_{a \in A} \overline{\lambda(a)} a$. $)$ Because $A$ is abelian, the idempotents $e_{\lambda}$ form a basis for $\mathbb{C}[A]$, and thus there exist coefficients $a_{\lambda} \in \mathbb{C}$ such that $v=\sum_{\lambda} a_{\lambda} e_{\lambda}$. If $\lambda$ and $\mu$ are distinct linear characters of $A$ and we view them (by linear extension) as being defined on the entire group algebra $\mathbb{C}[A]$, then $\lambda\left(e_{\lambda}\right)=1$ and $\mu\left(e_{\lambda}\right)=0$. It follows that

$$
a_{\lambda}=\lambda(v)=\sum_{g \in A} \lambda(g) f(g)=|A| \hat{f}(\bar{\lambda}),
$$

where the third equality follows from the definition of $\hat{f}$. We see, therefore, that $|\operatorname{supp}(\hat{f})|$ is equal to the number of coefficients $a_{\lambda}$ that are nonzero.

It is well known that the ideals of $\mathbb{C}[A]$ are exactly the subspaces of $\mathbb{C}[A]$ spanned by the various subsets of the set $\left\{e_{\lambda} \mid \lambda \in \hat{A}\right\}$. (This, of course, is because the group $A$ is abelian. The corresponding general statement for an arbitrary finite group $G$ is that the ideals of the center $\mathbf{Z}(\mathbb{C}[G])$ of the group algebra are the subspaces spanned by the central idempotents $e_{\chi}$, where $\chi$ runs over $\operatorname{Irr}(G)$, the set of irreducible characters of $G$.)

It follows that $|\operatorname{supp}(\hat{f})|$ is the dimension of the smallest ideal of $\mathbb{C}[A]$ that contains $v$. Since in this case the ideals of $\mathbb{C}[A]$ are precisely the $G$-submodules of $F[S]$, we conclude that $|\operatorname{supp}(\hat{f})|$ is equal to the dimension of the $G$-submodule $\langle v\rangle$ of $F[S]$ generated by $v$. We see, therefore, that the following result includes Theorem A.

Theorem B. Let $S$ be a finite transitive right $G$-set and let $0 \neq v \in F[S]$, where $F$ is an arbitrary field. Then

$$
|\operatorname{supp}(v)| \operatorname{dim}(\langle v\rangle) \geq|S|,
$$

where $\langle v\rangle$ is the $G$-submodule of $F[S]$ generated by $v$.

Observe that this result generalizes Theorem A in three distinct ways. The group $G$ need not be abelian and the field $F$ need not be the complex numbers. Also, the function $f$ need not be defined on the group $G$ itself; it can be defined instead on an arbitrary transitive right $G$-set $S$.

Our proof of Theorem B is quite easy, and in fact, it is shorter than the proof of Theorem A given in [12]. 
Proof of Theorem B. The submodule $\langle v\rangle \subseteq F[S]$ is the linear span of the $G$ translates $v g$ of $v$ as $g$ runs over $G$, and so we can choose a basis $\mathcal{B}$ for $\langle v\rangle$ consisting of such translates. Since $v$ is nonzero and $G$ acts transitively on $S$, we see that every point of $S$ is in $\operatorname{supp}(v g)$ for some element $g \in G$. But $v g$ is a linear combination of members of the basis $\mathcal{B}$, and thus $S=\bigcup_{b \in \mathcal{B}} \operatorname{supp}(b)$. It follows that $\sum_{b \in \mathcal{B}}|\operatorname{supp}(b)| \geq|S|$. If $b \in \mathcal{B}$, however, then $b$ is a translate of $v$, and we see that $|\operatorname{supp}(b)|=|\operatorname{supp}(v)|$. We conclude that $|\mathcal{B}||\operatorname{supp}(v)| \geq|S|$, and since $|\mathcal{B}|=\operatorname{dim}(\langle v\rangle)$, the proof is complete.

In order to facilitate discussion of Theorem $\mathrm{B}$ and related results, we establish the following notation. Given $v \in F[S]$, write $t=t(v)=|\operatorname{supp}(v)|$ and $d=d(v)=$ $\operatorname{dim}_{F}(\langle v\rangle)$, and set $n=|S|$. The assertion of Theorem B, therefore, is that if $t>0$, then $t d \geq n$.

It is not hard to describe exactly when equality holds in Theorem B, and we discuss this next. It is clear from our proof that if equality holds, then the supports of distinct members of the basis $\mathcal{B}$ must be disjoint. Since $\mathcal{B}$ can be chosen to contain any two linearly independent translates of $v$, it follows that if $\Delta=\operatorname{supp}(v)$ and $g \in G$, then either $\Delta g \cap \Delta=\emptyset$, or else $v g=a v$ for some nonzero scalar $a \in F$, and in particular, $\Delta g=\Delta$.

Recall that a nonempty subset $\Delta$ of the $G$-set $S$ is called a block if for each element $g \in G$, either $\Delta g=\Delta$ or $\Delta g \cap \Delta=\emptyset$. Of course, $\Delta=S$ is a block, and so is $\Delta=\{s\}$ for each point $s \in S$. These are the trivial blocks, and we recall that the transitive $G$-set $S$ is said to be primitive if every block is trivial. Observe that if $\Delta$ is a block, then so is every $G$-translate, and thus the distinct translates of $\Delta$ partition $S$. The number of such translates, therefore, is $|S| /|\Delta|$.

If equality holds in Theorem $\mathrm{B}$, we know that $\Delta=\operatorname{supp}(v)$ must be a block. Conversely, given any block $\Delta \subseteq S$, consider the vector $v=\sum_{x \in \Delta} x$. Then $\operatorname{supp}(v)=\Delta$, and in fact, the supports of the distinct $G$-translates of $v$ are exactly the $|S| /|\Delta|=n / t$ distinct translates of $\Delta$. The translates of $v$ are thus linearly independent, and we have $d=d(v)=n / t$, so equality holds in Theorem B.

It is possible to have $t d=n$ even if $v$ is not a scalar multiple of the sum of the points in its support. We digress briefly to discuss the most general possible case where this equality holds. Assuming equality, write $\Delta=\operatorname{supp}(v)$ and let $H=G_{\Delta}$ be the (setwise) stabilizer of the block $\Delta$. If $h \in H$, then the translate $v h$ also has support $\Delta$, and thus, as we have seen, $v h$ must be a scalar multiple of $v$. If we write $v h=\lambda(h) v$, where $\lambda(h) \in F$, it is easy to see that $\lambda$ is a homomorphism from $H$ into $F^{\times}$. Also, if $x \in \Delta$ and $K=G_{x}$ is the stabilizer of $x$, then $K \subseteq H$ since $\Delta$ is a block. If $k \in K$, we must have $\lambda(k)=1$ because the (nonzero) coefficients of $x$ in $v$ and in $v k=\lambda(k) v$ are equal.

In the situation of the previous paragraph, the vector $v$ is uniquely determined (up to a scalar multiple) by the block $\Delta$ and the homomorphism $\lambda$ from $H=G_{\Delta}$ into $F^{\times}$. To see this, fix $x \in \Delta$ and note that $x H=\Delta$. If the coefficient of $x$ in $v$ is $a$, then the coefficient of $x h$ in $v$ equals the coefficient of $x$ in $v h^{-1}=\lambda\left(h^{-1}\right) v$. Since this coefficient is $a \lambda\left(h^{-1}\right)$, it follows that $v$ is determined by $\Delta, \lambda$ and the scalar $a$, as claimed.

Conversely, let $\Delta$ be any block of the right $G$-set $S$. Write $H=G_{\Delta}$ and let $\lambda: H \rightarrow F^{\times}$be a homomorphism such that $G_{x} \subseteq \operatorname{ker} \lambda$ for $x \in \Delta$. We construct a corresponding vector $v \in F[S]$ as follows. Fix $x \in \Delta$ and recall that $x H=\Delta$. Write $v=\sum_{y \in \Delta} a_{y} y$, where $a_{x h}=\lambda\left(h^{-1}\right)$, and note that this is well defined 
because $G_{x} \in \operatorname{ker} \lambda$. It is easy to check that the supports of the translates of $v$ are exactly the $|S| /|\Delta|$ translates of $\Delta$, and that any two translates of $v$ with equal supports are scalar multiples of one another. It follows that $d=d(v)=|S| /|\Delta|$, and thus $t d=n$, as wanted.

If $S$ is a primitive $G$-set, then all blocks are trivial, and so if $1<t<n$, then equality cannot hold in Theorem B and we have $t d>n$. In fact, an even stronger inequality holds in the primitive case.

Theorem C. Let $S$ be a finite primitive right $G$-set and suppose that $v \in F[S]$, where $F$ is an arbitrary field. If $1 \leq t<n$, we have $(t+1) d \geq 2 n$, where as usual, $t=|\operatorname{supp}(v)|, d=\operatorname{dim}_{F}(\langle v\rangle)$ and $n=|S|$.

We shall see that equality can hold in Theorem C, but only under highly restrictive conditions.

Theorem D. Suppose that $(t+1) d=2 n$ in Theorem C, where $1<t<n-1$. Then $t=d$ and the action of $G$ on the set $\Omega$ of $G$-translates of $\operatorname{supp}(v)$ is doubly transitive. Furthermore, every point of $S$ lies in exactly two members of $\Omega$, and every two distinct members of $\Omega$ intersect in a single point. Finally, the field $F$ must have characteristic 2 and $v$ is a scalar multiple of the sum of the points in its support.

Note that in the situation of Theorem $\mathrm{D}$, the group $G$ is a doubly transitive permutation group in which the action on the collection of two-point subsets is primitive. Conversely, we shall see that given any such doubly transitive group and any field of characteristic 2, there is a corresponding example where equality holds in Theorem C. In Section 4, we describe exactly which doubly transitive groups act primitively on the two-point subsets.

If $n=|S|$ is a prime number, then the $G$-set $S$ is automatically primitive. In this case, and provided that the field $F$ has characteristic 0 , we prove an even better inequality than that of Theorem C.

Theorem E. Let $0 \neq v \in F[S]$, where $S$ is a transitive right $G$-set of prime cardinality $n$ and $F$ has characteristic 0 . Then $t+d>n$, where $t=|\operatorname{supp}(v)|$ and $d=\operatorname{dim}_{F}(\langle v\rangle)$.

We show in Section 6 that Theorem $\mathrm{E}$ is essentially equivalent to a certain theorem of N. G. Chebotarëv concerning complex roots of unity of prime order. We give an independent proof of Theorem E, and this, in turn, yields a new proof of Chebotarëv's result.

While this paper was in its final stages of preparation, the authors learned that what is essentially our Theorem E was independently (and approximately simultaneously) discovered by T. Tao. His preprint [11] presents a proof of Chebotarëv's result and deduces Theorem E from it. Also, Tao credits A. Biró with independent discovery of the same inequality.

The inequality $t+d>n$ of Theorem $\mathrm{E}$ is the best we can ever hope to prove under any set of hypotheses. This fact is a consequence of the following easy lemma.

Lemma F. Let $S$ be a finite transitive right $G$-set and suppose that $M \subseteq F[S]$ is an arbitrary nonzero $G$-submodule, where $F$ is an arbitrary field. Then there exists a nonzero vector $v \in M$ such that $t+d \leq n+1$, where $t, d$ and $n$ have their usual meanings. 
Proof. Since $n+1-\operatorname{dim}(M) \leq n=|S|$, we can choose a subset $X \subseteq S$ such that $|X|=n+1-\operatorname{dim}(M)$. Let $W \subseteq F[S]$ be the space of all vectors with support contained in $X$, and note that $\operatorname{dim}(W)=|X|$. We have $\operatorname{dim}(W)+\operatorname{dim}(M)=$ $n+1>\operatorname{dim}(F[S])$, and it follows that $W \cap M>0$. Let $v$ be a nonzero vector in this intersection. Then $\operatorname{supp}(v) \subseteq X$, and so $t=|\operatorname{supp}(v)| \leq|X|=n+1-\operatorname{dim}(M)$. Also, $\langle v\rangle \subseteq M$, and thus $d=\operatorname{dim}(\langle v\rangle) \leq \operatorname{dim}(M)$ and we have

$$
t+d \leq(n+1-\operatorname{dim}(M))+\operatorname{dim}(M)=n+1,
$$

as wanted.

We close this introduction by mentioning that there already is in the literature a generalization to nonabelian groups of the theorem of Donoho and Stark. (See Section 8 of [4].) This result, which we paraphrase somewhat, is due to P. Diaconis and M. Shahshahani. It is much weaker than our Theorem B, and it follows as a corollary of our result.

Corollary G. Let $f$ be a nonzero complex-valued function on an arbitrary finite group $G$. For each character $\chi \in \operatorname{Irr}(G)$, choose a complex representation $\mathcal{R}$ that affords $\chi$, and construct the matrix $M_{\chi}=\sum_{g \in G} f(g) \mathcal{R}(g)$. Let $\mathcal{X}$ be the set of characters $\chi \in \operatorname{Irr}(G)$ such that $M_{\chi}$ is not the zero matrix. Then

$$
|\operatorname{supp}(f)| \sum_{\chi \in \mathcal{X}} \chi(1)^{2} \geq|G| \text {. }
$$

Of course, the representation $\mathcal{R}$ is determined by the character $\chi$ only up to similarity, but this ambiguity does not affect whether or not $M_{\chi}=0$. Note that if $G$ is abelian, then the set $\mathcal{X}$ consists exactly of the complex conjugates of the linear characters in the support of the Fourier transform $\hat{f}$. We see, therefore, that Corollary $\mathrm{G}$ reduces to Theorem A in the abelian case.

Proof of Corollary G. Let $v \in \mathbb{C}[G]$ be the vector corresponding to the function $f$. Recall that $\mathbb{C}[G]$ is the direct sum of its minimal ideals, and these ideals correspond to the irreducible characters of $G$. If we extend the representation $\mathcal{R}$ affording $\chi$ to the whole group algebra by linearity, then $\mathcal{R}$ is exactly the projection map of $\mathbb{C}[G]$ onto the direct summand corresponding to $\chi$. Also, $M_{\chi}=\mathcal{R}(v)$, and so it follows that $v$ lies in the sum of the minimal ideals for which $M_{\chi} \neq 0$. This sum has dimension $\sum_{\chi \in \mathcal{X}} \chi(1)^{2}$, and it clearly contains the right ideal of $\mathbb{C}[G]$ generated by $v$. Since $\operatorname{supp}(f)=\operatorname{supp}(v)$, we now see that the result follows by Theorem B applied to the action of $G$ on itself by right multiplication.

\section{Primitive actions}

In this section we prove Theorem $\mathrm{C}$, which asserts the inequality $(t+1) d \geq 2 n$ in the case where $S$ is a primitive $G$-set and $1 \leq t<n$. Also in this section we study the case where $(t+1) d=2 n$ and we prove Theorem D.

The key to proving the inequality of Theorem $\mathrm{C}$ is Rudio's lemma 9], which also appears as 8.2 in Chapter I of [13]. Actually, we need only the following weak form of Rudio's lemma, which is valid even if $S$ is infinite. (Of course, for the purpose of this paper, we only need the finite case.)

(2.1) Lemma. Let $S$ be a primitive right $G$-set and let $X$ be an arbitrary nonempty proper subset of $S$. If $u, v \in S$ are distinct, then there exists some $G$-translate of $X$ that contains exactly one of $u$ and $v$. 
The full statement of Rudio's lemma asserts that if $G$ is finite, we can prescribe which one of $u$ or $v$ lies in a translate of $X$. As we shall see in Section 7, however, that conclusion does not necessarily hold for infinite groups.

Proof of Lemma 2.1. For $x, y \in S$, write $x \sim y$ if $x$ and $y$ lie in exactly the same collection of translates of $X$. This clearly defines an equivalence relation on $S$, and our goal, of course, is to show that $u \not v$.

Since $X$ is nonempty and proper in $S$, there exist inequivalent members of $S$, and so the equivalence class $\Delta$ of $u$ is proper in $S$. The action of $G$ permutes the equivalence classes, and it is clear that $\Delta$ is a block. By primitivity, therefore, $\Delta=\{u\}$, and thus $u \not v$, as wanted.

The conclusion of Lemma 2.1 is only slightly weaker than the full conclusion of Rudio's lemma, and we digress briefly to explain this. Suppose we know that some translate of $X$ contains exactly one of $u$ or $v$ and we wish to be able to specify that (say) $u$ is in a translate of $X$ and $v$ is not. We show that this is possible if we assume one additional piece of information: that $u g=v$ for some element $g \in G$ of finite order. Of course, this condition holds for finite groups $G$, and thus together with Lemma 2.1, the following argument provides an alternative proof of Rudio's lemma.

Suppose that some translate $Y$ of $X$ contains $v$ but not $u$ and assume that $v=u g$, where $g^{n}=1$ for some positive integer $n$. We show that an appropriate translate of $Y$ contains $u$ but not $v$. We have $u g=v \in Y$ but $u g^{n}=u \notin Y$, and it follows that there exists an integer $m$ such that $u g^{m} \in Y$ but $u g^{m+1} \notin Y$. Then $u \in Y g^{-m}$ but $v \notin Y g^{-m}$ because $v g^{m}=u g^{m+1} \notin Y$.

We now return to our main theme and establish the inequality $(t+1) d \geq 2 n$ when $1 \leq t<n$ and $S$ is a primitive finite $G$-set.

Proof of Theorem C. We use a refinement of the argument in the proof of Theorem B. Choose a basis $\mathcal{B}$ for $\langle v\rangle$ that consists of translates of $v$ and note that $\bigcup_{b \in \mathcal{B}} \operatorname{supp}(b)=S$. For each point $s \in S$, write $m(s)$ to denote the number of members $b \in \mathcal{B}$ such that $s \in \operatorname{supp}(b)$, and observe that $m(s) \geq 1$ for all $s \in S$. Since $|\mathcal{B}|=d$ and $|\operatorname{supp}(b)|=t$ for all $b \in \mathcal{B}$, we see that $t d=\sum_{s \in S} m(s)$. (This is because each side of this equation counts the number of ordered pairs $(b, s) \in \mathcal{B} \times S$ such that $s \in \operatorname{supp}(b)$.)

We claim that for each member $b \in \mathcal{B}$, there is at most one point $s \in \operatorname{supp}(b)$ such that $m(s)=1$. Assuming this for the moment, we see that there are at most $|\mathcal{B}|$ points $s \in S$ such that $m(s)=1$ and that for all other points $s \in S$, we have $m(s) \geq 2$. Then

$$
t d=\sum_{s \in S} m(s) \geq 2|S|-|\mathcal{B}|=2 n-d,
$$

and the desired inequality follows.

Suppose then, that there exists $b \in \mathcal{B}$ and distinct points $x, y \in \operatorname{supp}(b)$ such that $m(x)=1=m(y)$. Then $b$ is the only member of $\mathcal{B}$ for which either $x$ or $y$ is in the support. It follows that for each linear combination $c$ of vectors in $\mathcal{B}$, either the support of $c$ contains both $x$ and $y$ or neither of them, depending on whether or not $b$ appears with a nonzero coefficient in the expansion of $c$ in terms of $\mathcal{B}$.

Now if $w$ is any $G$-translate of $v$, we have $w \in\langle v\rangle$, and thus $w$ is a linear combination of vectors in $\mathcal{B}$. It is not the case, therefore, that $\operatorname{supp}(w)$ contains 
exactly one of $x$ and $y$. Writing $X=\operatorname{supp}(v)$, it follows that no translate of $X$ contains exactly one of $x$ and $y$. But $1 \leq t<n$, and thus $X$ is nonempty and proper in $S$, and since $S$ is primitive, this contradicts Lemma 2.1. The proof is now complete.

In fact, this argument gives some additional information, which we will exploit in the following section.

(2.2) Lemma. Suppose that equality holds in Theorem $\mathrm{C}$ and let $\mathcal{B}$ be any linearly independent set of translates of $v$. Then each point of $S$ is in the support of at most two members of $\mathcal{B}$. Also, the support of each member of $\mathcal{B}$ contains a point that is not in the support of any other member of this set.

Proof. Since $\mathcal{B}$ is part of some basis for $\langle v\rangle$ consisting of translates of $v$, it is no loss to assume that $\mathcal{B}$ is such a basis. Since we are assuming that $(t+1) d=2 n$, it follows that equality holds in $(*)$, in the proof of Theorem C. But we know that $m(s) \geq 1$ for all $s \in S$ and that $m(s)=1$ for at most one point in the support of each of the $d$ members of $\mathcal{B}$. We see, therefore, that equality forces $m(s)=1$ for exactly one point in the support of each member of $\mathcal{B}$ and $m(s)=2$ for all other points. This completes the proof.

\section{Equality in Theorem C}

When is it true that the equality $(t+1) d=2 n$ holds for some vector $v \in F[S]$, where $S$ is a primitive $G$-set? If $t=1$, then by Theorem B, we have $d=n$, and equality automatically holds. At the opposite extreme, when $t=n$, it is clear that equality is impossible except in the degenerate case where $n=1$. We will discuss the case where $t=n-1$ later, and so we assume for this section that $1<t<n-1$, and we work toward a proof of Theorem $\mathrm{D}$, which gives highly restrictive necessary conditions for equality to hold. As we shall see, these conditions turn out to be sufficient too.

(3.1) Lemma. Suppose that equality holds in Theorem $\mathrm{C}$ and let $X$ and $Y$ be distinct translates of $\operatorname{supp}(v)$. Then the stabilizer in $G$ of each point of $X \cap Y$ stabilizes the set $X \cup Y$.

Proof. Let $a$ and $b$ be translates of $v$ that are supported on $X$ and $Y$, respectively, and note that $a$ and $b$ are linearly independent since they have distinct supports. Suppose that $x \in X \cap Y$ and that $x g=x$, where $g \in G$. Then $x$ is in the support of $a, b$ and $a g$, and hence by Lemma 2.2, these three vectors cannot be linearly independent. We conclude that $a g$ is a linear combination of $a$ and $b$, and so $\operatorname{supp}(a g) \subseteq \operatorname{supp}(a) \cup \operatorname{supp}(b)$. Thus $X g \subseteq X \cup Y$, and similarly, $Y g \subseteq X \cup Y$. It follows that $(X \cup Y) g=X \cup Y$, as required.

We also need the following elementary combinatorial fact and an easy consequence.

(3.2) Lemma. Let $\Omega$ be a collection of subsets of some set $S$ and assume that each point of $S$ lies in exactly two members of $\Omega$. Say that a subcollection $\Lambda \subseteq \Omega$ is even if every point of $S$ lies in an even number of members of $\Lambda$. The following then hold:

(a) The intersection of any two even subcollections of $\Omega$ is even. 
(b) If $\Lambda \subseteq \Omega$ is even, then each member of $\Lambda$ is disjoint from each member $\Omega-\Lambda$.

Proof. Suppose that $\Lambda, \Delta \subseteq \Omega$ are even. Let $s \in S$ and suppose that the number of members of $\Lambda \cap \Delta$ that contain $s$ is nonzero. If $X$ and $Y$ are the two members of $\Omega$ that contain $s$, then at least one of $X$ or $Y$ is in both $\Lambda$ and $\Delta$. Thus since these collections are even, both $X$ and $Y$ lie in both $\Lambda$ and $\Delta$. Then $s$ lies in exactly two members of $\Lambda \cap \Delta$, establishing (a).

Now suppose that $s \in S$ lies in some member $X \in \Lambda$, where $\Lambda$ is even. Then both members of $\Omega$ that contain $s$ lie in $\Lambda$, and so no member of $\Omega-\Lambda$ can contain $s$. This proves (b).

(3.3) Lemma. Let $S$ be a finite primitive $G$-set and let $\mathcal{T}$ be a set of vectors in $F[S]$, where $F$ is an arbitrary field. Suppose that the members of $\mathcal{T}$ have distinct supports and that $\Omega=\{\operatorname{supp}(v) \mid v \in \mathcal{T}\}$ is transitively permuted by $G$. If every point of $S$ lies in exactly two members of $\Omega$, then every proper subset of $\mathcal{T}$ is linearly independent, and so the dimension of the linear span of $\mathcal{T}$ is either $|\mathcal{T}|$ or $|\mathcal{T}|-1$.

Proof. We can suppose that $\mathcal{T}$ is not linearly independent, and we choose a minimal dependent subset $\mathcal{T}_{0} \subseteq \mathcal{T}$. We can thus write $\sum a_{v} v=0$, where the sum runs over $v \in \mathcal{T}_{0}$ and all of the coefficients $a_{v} \in F$ are nonzero. It follows that no point of $S$ can lie in the support of exactly one vector $v \in \mathcal{T}_{0}$.

Let $\Omega_{0}=\left\{\operatorname{supp}(v) \mid v \in \mathcal{T}_{0}\right\}$. We have seen that each point of $S$ lies in either zero or two members of $\Omega_{0}$, and so by the language of Lemma 3.2, the collection $\Omega_{0}$ is even. Since $\Omega_{0}$ is nonempty, we can choose a minimal nonempty even subcollection $\Lambda \subseteq \Omega_{0}$.

Let $U=\bigcup \Lambda$, the union of all members of $\Lambda$. Suppose that $g \in G$ is an element such that $U g \neq U$, and observe that $\Lambda g \neq \Lambda$. Now $\Lambda g$ is even, and thus $\Lambda \cap \Lambda g$ is even by Lemma 3.2(a). But $\Lambda \cap \Lambda \mathrm{g}$ is proper in $\Lambda$, and it follows by the minimality of $\Lambda$ that $\Lambda \cap \Lambda g=\emptyset$. Thus no member of $\Lambda g$ is in $\Lambda$, and so by Lemma 3.2(b), we see that each member of $\Lambda g$ is disjoint from $U$. It follows that $U \cap U g=\emptyset$, and hence $U$ is a block.

Since each point of $S$ is in more than one member of $\Omega$, the members of $\Omega$ cannot be singleton sets, and hence the block $U$ is not a singleton. But $G$ is primitive on $S$, and so we must have $U=S$, and thus no member of $\Omega$ is disjoint from $U$. By Lemma 3.2(b) it follows that $\Lambda=\Omega$, and thus $\Omega_{0}=\Omega$. Then $\mathcal{T}_{0}=\mathcal{T}$, and hence every proper subset of $\mathcal{T}$ is linearly independent. The span of $\mathcal{T}$, therefore, has dimension $|\mathcal{T}|-1$, as wanted.

Recall that Theorem D asserts (among other things) that the group $G$ has a doubly transitive action in which the stabilizer of a two-point subset is a maximal subgroup. (In other words, its action on the two-point subsets is primitive.) In order to obtain the conclusion in Theorem D that the field $F$ must have characteristic 2 , we need to study such doubly transitive groups, and that is the purpose of the following lemma. This lemma will also be crucial in finding the examples where equality holds in Theorem $\mathrm{C}$.

(3.4) Lemma. Let $G$ be a doubly transitive permutation group on a set $\Omega$ and assume that the stabilizer of a two-point subset of $\Omega$ is a maximal subgroup of $G$. If $N$ is a minimal normal subgroup of $G$, then either $N$ is a nonabelian simple group or $|N|=3=|\Omega|$. 
The key to the proof of Lemma 3.4 is to show that $N$ must be primitive on $\Omega$, and then it follows by a standard argument that $N$ is either abelian or simple. In the abelian case, the conclusion that $|N|=3$ follows easily from the primitivity of $G$ on the two-point subsets of $\Omega$. It is true (but not quite trivial) that in general, a nonabelian minimal normal subgroup of a 2-transitive group must be primitive, and hence simple. (See page 202 of [1].) The primitivity of the action of $N$ on $\Omega$ is much easier to prove, however, in our case, where $G$ acts primitively on the two-point subsets. We have decided, therefore, to give the direct and elementary proof.

Proof of Lemma 3.4. Write $|\Omega|=r$ and observe that $r \geq 3$ since the stabilizer of a two-point subset is proper. Also, because $G$ is 2 -transitive on $\Omega$, we see that $N$ is transitive, and thus $N$ cannot fix a two-point subset of $\Omega$. Since the action of $G$ on these subsets is primitive and $N$ acts nontrivially, it follows that $N$ is transitive on the collection of two-point subsets, and this implies that $N$ is primitive on $\Omega$. (To see this, observe that if there were a nontrivial $N$-block $\Delta \subseteq \Omega$, we could choose $\alpha, \beta \in \Delta$ and $\gamma \in \Omega-\Delta$, and then no element of $N$ could take $\{\alpha, \beta\}$ to $\{\alpha, \gamma\}$.)

Since $N$ is minimal normal in $G$, we see that if it is not simple, we can write $N=A \times B$, where each of $A$ and $B$ is nontrivial. Since $N$ is primitive on $\Omega$, it follows that each of $A$ and $B$ is transitive, and since $A$ and $B$ centralize each other, we conclude that they are both regular. Thus $|A|=r=|B|$ and $|N|=r^{2}$.

Now let $K=G_{\alpha}$ be a point stabilizer in $G$ and note that $|K \cap N|$ is coprime to $r-1$ since $|N|=r^{2}$. Also, $K$ acts transitively on the $r-1$ points of $\Omega-\{\alpha\}$, and $K \cap N \triangleleft K$. It follows from this that $K \cap N$ acts trivially on $\Omega$. Then $K \cap N=1$, and so $N$ is regular and $|N|=r$, which is a contradiction. We conclude that $N$ is simple.

Finally, if $N$ is abelian, then it is regular and $|N|=r$. But $N$ acts transitively on the $r(r-1) / 2$ two-point subsets of $\Omega$, and thus $r \geq r(r-1) / 2$ and $r \leq 3$. We know that $r \geq 3$, however, and thus $r=3$, as required.

(3.5) Corollary. Let $G$ be a doubly transitive permutation group on a set $\Omega$ having at least four points, and assume that the stabilizer of a two-point subset of $\Omega$ is a maximal subgroup of $G$. If $1<N \triangleleft G$, then $N$ acts doubly transitively on $\Omega$.

Proof. As in the previous proof, $N$ acts nontrivially on the collection of two-point subsets of $\Omega$. Since $G$ is primitive on this collection and $N$ is a normal subgroup that acts nontrivially, it follows that $N$ acts transitively on the collection of twopoint subsets. To show that $N$ acts doubly transitively on $\Omega$, therefore, it suffices to show that some element of $N$ interchanges some pair of points of $\Omega$. By Lemma 3.4, however, $N$ is not solvable, and thus by the Feit-Thompson theorem, $N$ contains an element of order 2. Such an element, of course, interchanges a pair of points.

The following result is a somewhat more precise version of Theorem $\mathrm{D}$ of the Introduction.

(3.6) Theorem. Suppose that equality holds in Theorem $\mathrm{C}$ and that $1<t<n-1$. Let $\Omega$ be the set of $G$-translates of $\operatorname{supp}(v)$. The following then hold:

(a) Each point of $S$ lies in exactly two members of $\Omega$, and every two distinct members of $\Omega$ have exactly one point of $S$ in common.

(b) The action of $G$ on $\Omega$ is 2-transitive, and the induced action on two-element subsets of $\Omega$ is primitive. 
(c) $d=|\Omega|-1=t$.

(d) Each member of $\Omega$ is transitively permuted by its (setwise) stabilizer in $G$.

(e) $v$ is a scalar multiple of the sum of the points in its support.

(f) $F$ has characteristic 2 .

Proof. We have $(t+1) d=2 n$ and $2<t+1<n$, and thus $2<d<n$. In particular, since $d$ is a divisor of $2 n$, we see that $n$ cannot be prime. Also, since $G$ is primitive on $S$ and $|S|$ is not prime, it is easy to see that $G$ is generated by the stabilizers of any two distinct points of $S$.

Let $X, Y \in \Omega$ be distinct and write $u=|X \cap Y|$. We argue first that $u \leq 1$. Otherwise, let $x, y \in X \cap Y$ be distinct. By Lemma 3.1, the point stabilizers $G_{x}$ and $G_{y}$ both stabilize the set $X \cup Y$, and since these subgroups generate $G$, it follows that $G$ stabilizes $X \cup Y$. Then $X \cup Y=S$, and we have $n=2 t-u$.

Now let $Z, W \in \Omega$ be arbitrary. Then $Z \cup W \subseteq S=X \cup Y$, and since all members of $\Omega$ have equal cardinality, it follows that $|Z \cap W| \geq|X \cap Y| \geq 2$. The previous argument now shows that if $Z \neq W$, then $S=Z \cup W$, and thus $|Z \cap W|=|X \cap Y|=u$.

Since the set $X \cap Y$ is not $G$-invariant, it cannot be the case that $\Omega=\{X, Y\}$, and thus we can choose $Z \in \Omega$, different from $X$ and $Y$. Then $X \cup Z=S=X \cup Y$, and so $S-X \subseteq Y \cap Z$. Then $n-t=|S-X| \leq|Y \cap Z|=u$, and we have $2 t-u=n \leq t+u$, and thus $t \leq 2 u$. We have

$$
t d<(t+1) d=2 n=4 t-2 u \leq 3 t,
$$

and we conclude that $d<3$. Since $d>2$, this is a contradiction, and thus every two distinct members of $\Omega$ have at most one point in common.

Now let $X, Y, Z \in \Omega$ be distinct and suppose that $X \cap Y \cap Z$ is nonempty. Let $x \in X \cap Y \cap Z$ and observe that by the result of the previous argument, the intersection of any two of $X, Y$ and $Z$ is exactly the set $\{x\}$. Let $a, b$ and $c$ be translates of $v$ with supports $X, Y$ and $Z$, respectively, and note that by Lemma 2.2 , these three vectors cannot be linearly independent. Thus $c$ (say) is a linear combination of $a$ and $b$, and hence $Z \subseteq X \cup Y$. Then $Z-X \subseteq Y$, and we have $Z-X \subseteq Y \cap Z=\{x\}$. But $x \in X$, and it follows that $Z-X$ is empty. This is a contradiction since $|X|=|Z|$ and $X \neq Z$. We conclude that each point of $S$ lies in at most two members of $\Omega$.

The action of $G$ on $S$ is primitive, and since $1<t<n$, it follows that $\operatorname{supp}(v)$ is not a block. The members of $\Omega$, therefore, are not pairwise disjoint, and so some point of $S$ lies in two of them. Because $G$ is transitive on $S$, it follows that every point in $S$ lies in exactly two members of $\Omega$, and this defines a map from $S$ into the collection of two-element subsets of $\Omega$. This map is injective since distinct members of $\Omega$ can have at most one point in common. To complete the proof of (a), we must show that our map is surjective, and so it suffices to show that $r(r-1) / 2=n$, where $r=|\Omega|$. Since $2 n=(t+1) d$, we see that (a) will follow once we prove (c).

Since $|\Omega|=r$, we see that $2 n=t r$ because both sides of this equation count pairs $(x, X) \in S \times \Omega$ such that $x \in X$. Then $t r=(t+1) d$, and so $d<r$. If two translates of $v$ have the same support, then by Lemma 2.2, they must be scalar multiples of one another. It follows that $\langle v\rangle$ is spanned by a collection of translates of $v$, one supported on each of the $r$ members of $\Omega$. Since $d<r$, we see by Lemma 3.3 that $d=r-1$, as wanted. Then $(t+1) d=2 n=t r=t(d+1)$, and it follows that $t=d$. This establishes (c) and (a). 
We have a natural bijection between $S$ and the collection of two-member subsets of $\Omega$, and so we know that $G$ is transitive, and in fact primitive, on this collection. To show that $G$ is 2-transitive on $\Omega$ and thus complete the proof of (b), it suffices to show that if $x \in S$, then some element of $G_{x}$ interchanges the two members $X, Y \in \Omega$ that contain $x$. Certainly, $G_{x}$ permutes $\{X, Y\}$, and it suffices, therefore, to show that $G_{x}$ does not stabilize $X$. But if $G_{x} \subseteq G_{X}$, then since $G_{x}$ is maximal in $G$, we have $G_{x}=G_{X}$ and $\operatorname{tr} / 2=n=\left|G: G_{x}\right|=\left|G: G_{X}\right|=r$. Then $t=2$, and since we know that $t=d \neq 2$, we have a contradiction. This proves (b).

Let $X \in \Omega$. To prove (d), we must show that $G_{X}$ is transitive on $X$. But the points of $X$ are exactly the intersections of $X$ with the various members of $\Omega-\{X\}$, and since $G$ is 2-transitive on $\Omega$, these sets are transitively permuted by $G_{X}$, and thus the points of $X$ are also transitively permuted by $G_{X}$, as wanted.

For (e), let $X=\operatorname{supp}(v) \in \Omega$ and write $K=G_{X}$. We know by Lemma 2.2 that $v k$ must be a scalar multiple of $v$ for each element $k \in K$, and we define the map $\lambda: K \rightarrow F$ by $v k=\lambda(k) v$. Observe that $\lambda$ is a homomorphism from $K$ into $F^{\times}$.

Let $A$ be a set of representatives for the right cosets of $K$ in $G$ and note that the $r=d+1$ vectors $v a$ for $a \in A$ have distinct supports. Also, every translate of $v$ is a scalar multiple of one of the vectors $v a$ with $a \in A$, and hence these vectors span $\langle v\rangle$, which has dimension $d$. It follows that there is a unique (up to scalar multiplication) linear dependence relation among the vectors $v a$, and we write $\sum_{a \in A} c_{a} v a=0$, where the coefficients $c_{a}$ lie in $F$. Also, we know from Lemma 3.3 that every proper subset of $\{v a \mid a \in A\}$ is linearly independent, and thus $c_{a} \neq 0$ for all $a \in A$.

Let $g \in G$ be arbitrary and let $a \in A$. Write $a \cdot g$ to denote the unique element of $A$ that lies in the coset $K a g$. Now apply $g$ to the equation $\sum c_{a} v a=0$ and express each vector $v a g$ as an appropriate scalar multiple of $v(a \cdot g)$. (In fact, it is easy to see that this scalar is $\lambda\left(a g(a \cdot g)^{-1}\right)$, although we shall not need this explicit formula.) Since the map $a \mapsto a \cdot g$ is a permutation of $A$, what results is a new dependence relation of the form $\sum b_{a} v a=0$. It follows that there must exist a scalar $\mu(g)$, depending only on $g$, such that $b_{a}=\mu(g) c_{a}$ for all $a \in A$. Furthermore, it is not hard to see that $\mu: G \rightarrow F^{\times}$is a group homomorphism. (What is really going on here is that $F v$ is a 1 -dimensional $K$-module, and the vector $\sum_{a \in A} c_{a}(v \otimes a)$ spans a 1-dimensional $G$-submodule of the induced module $(F v)^{G}$.)

We can suppose that $1 \in A$. If we take $g \in K$, then $1 \cdot g=1$, and since $v g=\lambda(g) v$, we see that $b_{1}=\lambda(g) c_{1}$, and thus $\mu(g)=\lambda(g)$ and $\mu$ is an extension of $\lambda$ to $G$. (This conclusion could also be proved using an appropriate generalization of Frobenius reciprocity.)

Write $N=\operatorname{ker} \mu \triangleleft G$ and observe that $N>1$ since it is clear that $G$ is noncyclic, and thus $N$ is doubly transitive on $\Omega$ by Corollary 3.5. Also, $N \cap K=\operatorname{ker} \lambda$, and this is exactly the stabilizer of $v$ in $K$, and hence it is the stabilizer of $v$ in $G$.

Let $x \in X$ and write $H=G_{x}$. If $g \in H \cap K$, then $v g=\lambda(g) v$ and the (nonzero) coefficients of $x$ in $v$ and $v g$ are equal. It follows that $\lambda(g)=1$, and thus $\mu(g)=1$ and $g \in N$. This shows that $H \cap K \subseteq N$.

Now $H \cap K$ stabilizes both $X$ and the unique other member of $\Omega$ that contains $x$. In fact, $H \cap K$ is a full two-point stabilizer in the 2-transitive action of $G$ on $\Omega$, and so $|G: H \cap K|=r(r-1)$. If $N<G$, then $|N: H \cap K|<r(r-1)$, and thus $N$ is not 2-transitive on $\Omega$, a contradiction. We conclude, therefore, that $N=G$, and 
thus $K=N \cap K$ stabilizes $v$. By (d), however, $K$ is transitive on $X=\operatorname{supp}(v)$, and (e) follows.

We can replace $v$ by a scalar multiple and assume that $v$ is exactly the sum of the points in its support. For each member $X \in \Omega$, write $v_{X}=\sum_{x \in X} x$, and note that these $r=|\Omega|$ vectors are exactly the $G$-translates of $v$. But $d<r$, and so the vectors $v_{X}$ are dependent, and we can write $\sum_{X \in \Omega} c_{X} v_{X}=0$ for suitable coefficients $c_{X} \in F$, not all 0 .

Choose distinct members $X, Y, Z \in \Omega$ with $c_{X} \neq 0$. There is a point of $S$ that lies in $X$ and $Y$ and in no other member of $\Omega$, and it follows that $c_{X}+c_{Y}=0$. Similarly, $c_{X}+c_{Z}=0$ and $c_{Y}+c_{Z}=0$, and it follows that $2 c_{X}=0$. We deduce that $F$ has characteristic 2, as required for (f). The proof is now complete.

To summarize, we see that if equality holds in Theorem $\mathrm{C}$ and $1<t<n-1$, then $G$ is a 2 -transitive group on some set $\Omega$ such that the induced action on the two-point subsets of $\Omega$ is primitive. Also, $t=d$, and thus $2 n=t(t+1)$. It follows that we cannot have $t=2$ since otherwise $n=3$ and the inequality $t<n-1$ would not hold. Thus $t \geq 3$, and so $|\Omega|=t+1 \geq 4$.

It is possible (by appealing to the classification of finite simple groups) to list all possible doubly transitive groups $G$ on four or more points such that the action of $G$ on the two-point subsets is primitive. We shall see in the next section that given any such group and any field of characteristic 2, it is possible to construct a corresponding example where equality holds in Theorem C.

\section{The EXAmples Where $1<t<n-1$}

We know that if equality holds in Theorem $\mathrm{C}$ and $1<t<n-1$, then $G$ has a doubly transitive permutation representation on some set $\Omega$ of cardinality $t+1$, where the induced action on two-point subsets of $\Omega$ is primitive.

Conversely, suppose that $G$ is doubly transitive on some set $\Omega$ with $|\Omega| \geq 3$. Let $S$ be the set of two-point subsets of $\Omega$ and suppose that the action of $G$ on $S$ is primitive. Let $K$ be the stabilizer in $G$ of a point $\alpha \in \Omega$ and let $H$ be the stabilizer in $G$ of the two-point set $\{\alpha, \beta\} \subseteq S$.

Since $G$ is doubly transitive on $\Omega$, some element $t \in G$ interchanges $\alpha$ and $\beta$, and we see that $t \in H$, and so $H$ is transitive on $\{\alpha, \beta\}$. It follows that $|H: H \cap K|=2$. Also, $K$ is transitive on $\Omega-\{\alpha\}$, which contains at least 2 points, and so $K$ does not fix $\beta$, and it follows that $K \nsubseteq I H$. Finally, we observe that since $|\Omega|>2$, we have $|S|>1$, and so $K$, which stabilizes a point of $S$, is certainly not transitive on $S$.

We consider a somewhat more general situation.

(4.1) Lemma. Let $S$ be a finite primitive right $G$-set and let $H=G_{x}$ for some point $x \in S$. Suppose that $K \subseteq G$ is any subgroup that satisfies the following three conditions:

(1) $|H: H \cap K|=2$.

(2) $K \nsubseteq H$.

(3) $K$ is not transitive on $S$.

Let $F$ be any field, and let $v \in F[S]$ be the sum of the points in the $K$-orbit of $x$. As usual, write $t=t(v), d=d(v)$ and $n=|S|$. The following then hold:

(a) $t=|K: H \cap K|$.

(b) $t|G: K|=2 n$. 
(c) Either $d=|G: K|$ or $d=|G: K|-1$.

(d) If $F$ has characteristic 2 , then $d=|G: K|-1$.

Proof. Statement (a) is clear since $|K: H \cap K|$ is the size of the $K$-orbit of $x$. Thus $t|G: K|=|G: H \cap K|=2|G: H|=2 n$, where the second equality follows by assumption (1), which establishes (b). We work next to determine $d$.

We claim that $K$ is the full stabilizer in $G$ of the $K$-orbit $X$ containing $x$. Otherwise, there exists a subgroup $J \subseteq G$ such that $J$ stabilizes $X$ and $K<J$. In fact, $J<G$ since $X$ is proper in $S$ by assumption (3). Since $K$ is transitive on $X$ we have $J=K J_{x}=K(H \cap J)$, and thus $H \cap J \nsubseteq K$. Then $H \cap K<H \cap J \subseteq H$. But $|H: H \cap K|=2$, and so it follows that $H \cap J=H$ and $H \subseteq J$. In fact, $H<J$ since $K \subseteq J$ but $K \nsubseteq H$ by assumption (2). We now have $H<J<G$, and this is a contradiction since by the primitivity of $S$, the point stabilizer $H$ is maximal in $G$.

Let $\Omega$ be the set of $G$-translates of $X$. Then $|\Omega|=|G: K|$ since we now know that $G_{X}=K$. Since $G$ is transitive on $S$ and on $\Omega$, we see that the number of members of $\Omega$ that contain each point $y \in S$ is some constant $m$, independent of the choice of $y$. If we count ordered pairs $(y, Y)$, where $Y \in \Omega$ and $y \in Y$, we see that $m n=t|\Omega|=t|G: K|$, and thus $m=2$ by (b). Each point of $S$, therefore, lies in exactly two members of $\Omega$.

The translates of $v$ are exactly the $|G: K|$ vectors $v_{Y}=\sum_{y \in Y} y$ for $Y \in \Omega$, and $d$ is the dimension of the linear span of these vectors. By Lemma 3.3, therefore, either $d=|G: K|$ or $d=|G: K|-1$, proving (c).

If $F$ has characteristic 2, it follows from the fact that each point of $S$ lies in exactly two members of $\Omega$ that $\sum v_{Y}=0$, and thus the vectors $v_{Y}$ are dependent and we have $d<|G: K|$. Then $d=|G: K|-1$, as wanted.

In the situation of Lemma 4.1, write $r=|G: K|$. Then $d=r$ or $d=r-1$ and $t r=2 n$. Thus either $t d=2 n$ or $t(d+1)=2 n$, and if $F$ has characteristic 2 , then only the second alternative can occur.

In the case where $G$ is doubly transitive on $\Omega$ and $H$ is the stabilizer of a twopoint subset, we have $r=|\Omega|$, and thus $n=(r-1) r / 2$. If $F$ has characteristic 2 , then $d=r-1$ and $t(d+1)=2 n=(r-1) r=d(d+1)$, and so $t=d$. Then $(t+1) d=2 n$, and we have equality in Theorem C. Note also that if $|\Omega| \geq 4$, then $t=r-1 \geq 3$, and we certainly have $1<t<n-1$.

We now address the question of finding all doubly transitive permutation groups $G$ acting on a set $\Omega$ consisting of at least four points and such that the induced action of $G$ on the two-point subsets of $\Omega$ is primitive.

First, recall Lemma 3.4, which asserts that a minimal normal subgroup $N$ of $G$ must be a nonabelian simple group. By Corollary 3.5, furthermore, the simple group $N$ must itself be doubly transitive on $\Omega$, and so $G$ is contained between $N$ and its normalizer in the symmetric group $S_{\Omega}$. (Note that since $N$ clearly has a trivial centralizer in $S_{\Omega}$, its normalizer, which we denote $\operatorname{Aut}_{\Omega}(N)$, is naturally embedded in $\operatorname{Aut}(N)$, and so can be computed.)

To find the examples we seek, therefore, we start by examining the list of doubly transitive simple groups, as compiled by P. Cameron [3]. For each such simple group $N$, acting doubly transitively on a set $\Omega$, we compute $A=\operatorname{Aut}_{\Omega}(N)$ and we check the groups $G$ with $N \subseteq G \subseteq A$ to determine which of them (if any) act primitively on the two-point subsets of $\Omega$. Once we find such a group $G$, then of course, any larger subgroup (contained in $A$ ) will also yield an example. 
The following table lists all simple groups $N$ that occur as minimal normal subgroups of doubly transitive groups that are primitive on the set of two-point subsets. In most of these cases, the simple group $N$ is itself primitive on the two-point subsets, and so every group contained between $N$ and $\operatorname{Aut}_{\Omega}(N)$ is an example, as desired. The only exceptions are $P S L(2, q)$ acting on $q+1$ points, where $q \in\{7,9,11\}$. These three simple groups do not act primitively on the twopoint subsets, but the overgroup $P G L(2, q)$ does have this property. (For $q=9$, the overgroup $M_{10}$ provides another example.)

\begin{tabular}{|c|c|c|}
\hline Simple group & Degree & Remarks \\
\hline \hline$A_{r}$ (Alternating) & $r$ & $r \geq 5$ \\
\hline$M_{r}$ (Mathieu) & $r$ & $r \in\{11,12,22,23,24\}$ \\
\hline$P S L(2, q)$ & $q+1$ & $q \notin\{2,3,5\}$ \\
\hline$S z(q)$ (Suzuki) & $q^{2}+1$ & $q=2^{2 k+1 \quad k \geq 1}$ \\
\hline$P S L(2,11)$ & 11 & \\
\hline$M_{11}$ (Mathieu) & 12 & \\
\hline$H S$ (Higman-Sims) & 176 & \\
\hline$C o_{3}$ (Conway) & 276 & \\
\hline
\end{tabular}

We will not actually prove that this table is complete, and we give only a partial proof that it is correct. First (and this does not depend on the classification of simple groups), almost every group that is triply (and not just doubly) transitive and that fails to have an abelian normal subgroup is guaranteed to act primitively on two-point subsets. (The exception here is the action of the symmetric group $S_{5}$ on 6 points.) This justifies the appearance in our table of the alternating groups and the Mathieu groups in their natural permutation representations. It covers the groups $P S L(2, q)$, where $q$ is a power of 2 exceeding 2 , and also it shows that $M_{11}$ in its 12-point representation belongs in our table.

This fact about triply transitive groups is an easy corollary of the following theorem of Cameron [2].

(4.2) Theorem (Cameron). Suppose that $G$ is a triply transitive permutation group on a set $\Omega$ and let $\alpha \in \Omega$. Assume that $G$ also acts on a set $\Lambda$ in such a way that $\Omega-\{\alpha\}$ and $\Lambda$ are isomorphic as $G_{\alpha}$-sets. Then either $G$ has a nontrivial abelian normal subgroup or else $G \cong S_{5}$ and $|\Omega|=6$.

(4.3) Corollary. Let $G$ be a triply transitive permutation group on a set $\Omega$ and assume that $G$ has no nontrivial abelian normal subgroup. Then the action of $G$ on the two-point subsets of $\Omega$ is primitive unless $G \cong S_{5}$ and $|\Omega|=6$.

Proof. Let $\alpha, \beta \in \Omega$ and (as usual) let $K=G_{\alpha}$ and $H=G_{\{\alpha, \beta\}}$. Assuming that the action of $G$ on the two-point subsets of $\Omega$ is not primitive, the subgroup $H$ is not maximal, and we let $H<J<G$. Since $G$ is triply transitive, we see that $\{\alpha, \beta\}$ and $\Omega-\{\alpha, \beta\}$ are the orbits of $H$ on $\Omega$. But $J$ does not stabilize the set $\{\alpha, \beta\}$ and $J>H$, and it follows that $J$ is transitive on $\Omega$ and we have $J K=G$.

Since $J<G$, we see that $K \nsubseteq J$, and thus $H \cap K \subseteq J \cap K<K$. But $K$ acts doubly transitively, and hence primitively on $\Omega-\alpha$, and $H \cap K$ is the stabilizer of 
the point $\beta$ in this action. It follows that $H \cap K$ is maximal in $K$ and we conclude that $H \cap K=J \cap K$.

Let $\Lambda$ be the set of right cosets of $J$ in $G$. Then $K$ acts transitively on $\Lambda$ since $J K=G$, and $K \cap J=K \cap H$ is the stabilizer of a point in this action. Also, $K$ acts transitively on $\Omega-\{\alpha\}$, and $K \cap H$ is the stabilizer of a point in that action. It follows that $\Lambda$ and $\Omega-\{\alpha\}$ are isomorphic as $K$-sets, and the result follows via Cameron's theorem.

Finally, we explain why the group $\operatorname{PSL}(2, q)$ appears in our table when $q>11$ is odd. Consider the action of $S L(2, q)$ on the $q+1$ subspaces of dimension 1 in a 2-dimensional space $V$ over the field $F$ of order $q$. If $\alpha=F v$ and $\beta=F w$ are distinct "points" and we use $\{v, w\}$ as our basis for $V$, then the stabilizer in $S L(2, q)$ of the set $\{\alpha, \beta\}$ is easily seen to be the group of monomial matrices of determinant 1. This group is dihedral of order $2(q-1)$, and it corresponds to a dihedral subgroup of order $q-1$ in $\operatorname{PSL}(2, q)$. Since $q>11$, we see that neither $A_{5}$ nor $S_{4}$ contains a dihedral subgroup of order $q-1$, and it follows by checking the list of isomorphism types of subgroups of $\operatorname{PSL}(2, q)$ that a dihedral subgroup of order $q-1$ is necessarily maximal. (See 6, Hauptsatz II.8.27].) In other words, the action of $\operatorname{PSL}(2, q)$ on the two-point subsets of the projective line over $F$ is primitive, as claimed.

We close this section with another application of Lemma 4.1. We know by Theorem $\mathrm{C}$ that $(t+1) d \geq 2 n$ in the primitive case, and we have discussed when it can happen that $(t+1) d=2 n$. Lemma 4.1 can also be used to construct other examples where the quantity $t d$ is fairly small when compared with $n$. For example, suppose $G=P G L(2, p)$, where $p$ is a prime congruent to 3 modulo 4 and congruent to \pm 1 modulo 5 . Then $G$ has a maximal subgroup $H$ isomorphic to the symmetric group $S_{4}$ and also a subgroup $K$ isomorphic to the alternating group $A_{5}$ and such that $|H: H \cap K|=2$. By Lemma 4.1, this yields an example with $t=|K: H \cap K|=5$ and $2 n=5 r$, where $n=|G: H|$ and $r=|G: K|$. By Lemma 4.1 , we have $d \leq r$, and so $t d \leq 5 r=2 n$.

\section{THE CASE $t=n-1$}

In this section, we consider the case $t=n-1$ to conclude our analysis of equality in Theorem C. Since we are assuming that $(t+1) d=2 n$, we see that $d=2$ in this situation, and that the permutation module $F[S]$ has a 2-dimensional submodule. The following is an easy corollary of our Theorem C.

(5.1) Corollary. Let $S$ be a primitive right $G$-set and suppose that $M \subseteq F[S]$ is a 2-dimensional submodule. If $v \in M$ is nonzero, then either $t(v)=n$ or $t(v)=n-1$, where $n=|S|$. Also, if $t(v)=n-1$, then $d(v)=2$.

Proof. Write $t=t(v)>0$ and note that $d=d(v) \leq 2$ since $\langle v\rangle \subseteq M$. By Theorem $\mathrm{C}$, we have $2(t+1) \geq(t+1) d \geq 2 n$, and thus $t+1 \geq n$, as required. Also, if $t=n-1$, we have $d \geq 2$, and thus $d=2$.

It is clear that in the situation of Corollary 5.1 some nonzero element $v \in M$ satisfies $t(v)<n$, and thus $t(v)=n-1$ and $d(v)=2$. Whenever $F[S]$ has a 2-dimensional submodule, therefore, we are in a situation where equality holds in Theorem C. We proceed to classify the primitive permutation groups $G$ for which the permutation module $F[S]$ has a submodule of dimension 2. (For the sake 
of brevity, we will not carry out this classification for all fields $F$, but only for sufficiently large fields of some given characteristic.)

(5.2) Theorem. Let $S$ be a faithful primitive right $G$-set and let $H$ be the stabilizer of a point in the action of $G$ on $S$. Suppose that the permutation module $F[S]$ has a submodule of dimension 2, where $F$ is a field. Then $H$ is cyclic and $G$ has a normal elementary abelian q-subgroup $E$ for some prime $q$. Also, $E H=G$ and $H$ acts faithfully and irreducibly on E. Finally, if the prime $q$ is not the characteristic of $F$, then $|E|=q$ and $|H| \leq 2$.

Proof. Let $M \subseteq F[S]$ be a 2-dimensional $G$-submodule. As we have observed, $M$ must contain a vector $v$ with $t(v)=n-1$, where $n=|S|$, and we write $S-\operatorname{supp}(v)=\{x\}$. It follows that the stabilizer in $G$ of $v$ is contained in $G_{x}$, and in particular, $\operatorname{ker} M \subseteq G_{x}$. But $G_{x}$ contains no nontrivial normal subgroup of $G$, and thus $M$ is a faithful $G$-module.

Suppose now that some element $g \in G$ fixes two distinct points $y, z \in S$. We argue that $g=1$, and thus the action of $G$ on $S$ is either regular or Frobenius. If $w \in M$ is arbitrary, then $y, z \notin \operatorname{supp}(w-w g)$, and thus $t(w-w g) \leq n-2$. By Corollary 5.1, therefore, $w-w g=0$, and thus $g$ acts trivially on $M$. But $M$ is faithful, and thus $g=1$, as claimed.

The action of $G$ on $S$ is primitive, and so if it is regular, then $|G|$ is prime, and there is nothing further to prove. We can thus assume that the action of $G$ on $S$ is Frobenius, and hence there is a regular normal subgroup $E$ by Frobenius' theorem. Also, $E$ is nilpotent by Thompson's theorem, and it follows by primitivity that $E$ is an elementary abelian $q$-group for some prime $q$. Also, $G=E H$ and $H$ acts faithfully and irreducibly on $E$.

If $q$ is not the characteristic of $F$, then by standard properties of Frobenius groups, it follows that no faithful $G$-module can have dimension smaller than $|H|$. Thus $2=\operatorname{dim}(M) \geq|H|$, and so $|H|=2$. Since $H$ acts irreducibly on $E$, it follows that $|E|=q$, as wanted.

Finally, suppose that $q$ is the characteristic of $F$. Since the normal $q$-subgroup $E$ is not contained in $\operatorname{ker} M$, it follows that $M$ is not a simple $G$-module, and in particular, $M$ is not simple as an $H$-module. But $M$ is completely irreducible as an $H$-module by Maschke's theorem, since $q$ does not divide $|H|$. (This is because in a Frobenius group, the orders of the kernel and the complement are always coprime.) It follows that the restriction $M_{H}$ is the direct sum of two 1-dimensional submodules, and since $M$ is faithful, we see that $H$ is abelian. An abelian Frobenius complement, however, is necessarily cyclic, and this completes the proof.

All of the possibilities allowed by Theorem 5.2 can actually occur. To see this, we consider first the situation where $q$ (the prime divisor of the regular normal subgroup $E$ ) is not the characteristic of $F$. Then $G$ is either cyclic of order $q$ or dihedral of order $2 q$ and $|S|=q$. In this case, $F[S]$ is the direct sum of a trivial module and a module $V$ of dimension $q-1$. If $F$ is large enough to contain a primitive $q$ th root of unity, it is clear that a simple submodule of $V$ has dimension 1 or 2 , and in either case, $F[S]$ has a 2-dimensional submodule.

The more interesting case is where $q$ is the characteristic of $F$. In this situation, we have $G=H E$, where $E$ is an elementary abelian $q$-group, $H$ is cyclic and $H$ acts faithfully and irreducibly on $E$. We choose the field $F$ so that $|F|=|E|$, and 
we note that there is a subgroup $A \subseteq F^{\times}$with $H \cong A$. In this situation, we can identify $G$ with the group described in the following lemma.

(5.3) Lemma. Let $F$ be any field. If $a, b \in F$, with $a \neq 0$, let $g_{a, b}: F \rightarrow F$ be the affine linear map defined by $x \mapsto a x+b$. Now let $A \subseteq F^{\times}$be a subgroup. Then the set $G=\left\{g_{a, b} \mid a \in A, b \in F\right\}$ is a transitive permutation group on $F$. Also, $G$ is primitive if no nonzero proper subgroup of $F^{+}$is invariant under multiplication by A.

Proof. We compute that $(x) g_{a, b} g_{c, d}=(a c) x+(b c+d)$, and it follows easily that $G$ is a group of permutations of $F$. Since $g_{a, b}$ carries $0 \in F$ to $b$, and $b \in F$ is arbitrary, we see that the action of $G$ on $F$ is transitive.

The stabilizer in $G$ of the point $0 \in F$ is the subgroup $G_{0}=\left\{g_{a, 0} \mid a \in A\right\}$. If $G_{0} \subseteq H \subseteq G$, where $H$ is a subgroup, we let $K=\left\{t \in F \mid g_{1, t} \in H\right\}$. Then $K$ is a subgroup of $F^{+}$, and it is easy to see that $H=G$ if $K=F$ and $H=G_{0}$ if $K=0$. Also, if $b \in K$ and $a \in A$, then $a b \in K$, and therefore, if no nonzero proper subgroup of $F^{+}$is invariant under multiplication by $A$, it follows that $G_{0}$ is maximal in $G$, and so $G$ is primitive on $F$.

In our situation, $H$ acts irreducibly on $E$, and so in the language of Lemma 5.3, the action of $G$ on $F$ is primitive, and we take $S=F$. We show in this situation that the permutation module $F[S]$ has a 2-dimensional $G$-submodule.

To avoid confusion, we distinguish $F$ from $S$ by writing $s_{x} \in S$ to denote the element corresponding to $x \in F$. Take $v=\sum_{x \in F} x s_{x} \in F[S]$ and note that $t=t(v)=n-1$, where $n=|S|=|F|$. Also, let $w \in F[S]$ be the sum of the elements of $S$. We compute that

$$
v g_{a, b}=\sum_{x \in F} x s_{a x+b}=\sum_{y \in F} \frac{y-b}{a} s_{y}=\frac{1}{a}(v-b w),
$$

and thus $\langle v\rangle$ is contained in the subspace spanned by $v$ and $w$. Also, the above calculation shows that $w \in\langle v\rangle$, and since it is clear that $v$ and $w$ are linearly independent, we have $d=2$, and thus $\langle v\rangle$ is the desired 2-dimensional submodule.

\section{Prime Degree}

In this section we establish Theorem $\mathrm{E}$, which asserts the inequality $t+d>p$ in the case where $|S|=p$ is prime and the field $F$ has characteristic 0. As usual, $v \in F[S]$ is nonzero, $t=t(v)=|\operatorname{supp}(v)|$ and $d=d(v)=\operatorname{dim}_{F}(\langle v\rangle)$.

We shall see that the inequality of Theorem $\mathrm{E}$ is intimately related to the following theorem of N. G. Chebotarëv.

(6.1) Theorem (Chebotarëv). Let $p$ be prime and suppose that $\zeta \in \mathbb{C}$ is a primitive pth root of unity. Let $V$ be the Vandermonde matrix with $(i, j)$-entry equal to $\zeta^{i j}$, for $0 \leq i, j \leq p-1$. Then all square submatrices of $V$ have nonzero determinant.

Chebotarëv's proof of this result is presented by P. Stevenhagen and H. W. Lenstra in their expository paper [10]. There are also several other proofs of Chebotarëv's result in the literature, and four of these are referenced in [10]. Yet another proof, which we cannot resist mentioning, can be found in [5].

It is possible to interpret Theorem 6.1 in the spirit of the kinds of inequalities we are considering in this paper. To see how to do this, let $G=\langle z\rangle$ be a group of prime order $p$. The group of linear characters $\hat{G}$ of $G$ is also cyclic of order $p$, and 
we choose a generating character $\mu$. Write $\mu(z)=\zeta$ and note that $\zeta$ is a primitive $p$ th root of unity.

What would it mean to say that some square submatrix of the Vandermonde matrix $\left[\zeta^{i j}\right]$ has determinant 0 ? Clearly, this is equivalent to the existence of subsets $X, Y \subseteq\{0,1, \ldots, p-1\}$, where $|X|=|Y|$, and coefficients $a_{x}$ for $x \in X$, not all of them zero, such that $\sum_{x \in X} a_{x} \zeta^{x y}=0$ for all $y \in Y$. If we write $v=\sum_{x \in X} a_{x} z^{x} \in$ $\mathbb{C}[G]$, we see that this system of equations can be rewritten as $\mu^{y}(v)=0$ for $y \in Y$. In other words, the failure of Chebotarëv's assertion would be equivalent to the existence of some nonzero vector $v \in \mathbb{C}[G]$ such that $\lambda(v)=0$ for at least $t$ linear characters $\lambda$, where $t=|\operatorname{supp}(v)|$.

We saw in Section 1 that if $v \in \mathbb{C}[A]$, where $A$ is an abelian group, then the quantity $d=\operatorname{dim}(\langle v\rangle)$ is exactly equal to the number of linear characters $\lambda$ of $A$ such that $\lambda(v) \neq 0$, and so there are exactly $p-d$ linear characters $\lambda$ of $A$ such that $\lambda(v)=0$. The failure of Chebotarëv's assertion, therefore, would be equivalent to the existence of a nonzero vector $v \in \mathbb{C}[G]$ such that $p-d \geq t$. In other words, Theorem 6.1 implies that the inequality $t+d>p$ holds when $S=G$ has prime order $p$ and $F=\mathbb{C}$. Conversely, we see that if we could find an independent proof of this inequality, that would yield a proof of Chebotarëv's result. (We will present just such a proof later in this section.)

Finally, we mention that the equivalence that we have just established between the inequality $t+d>p$ and Chebotarëv's assertion is valid for any field that contains a primitive $p$ th root of unity. As we shall see, however, our inequality can fail for some such fields, and it follows that for those fields, the conclusion of Chebotarëv's theorem is false.

To prove Theorem E (assuming Chebotarëv's result) we reduce the general problem to the case where $S=G$ has prime order $p$ and $F=\mathbb{C}$. We need the following easy observation.

(6.2) Lemma. Let $S$ be a finite set and suppose that $F \subseteq E$ are fields. Let $V \subseteq F[S]$ be an $F$-subspace and write $E V$ to denote the E-span of $V$ in $E[S]$. Then $\operatorname{dim}_{E}(E V)=\operatorname{dim}_{F}(V)$.

Proof. An $F$-basis $\mathcal{B}$ for $V$ clearly spans $E V$ over $E$, and so it suffices to observe that $\mathcal{B}$ is linearly independent over $E$. This follows via elementary linear algebra, however, since a homogeneous system of linear equations with coefficients in $F$ that has a nontrivial solution over $E$ must also have a nontrivial solution over $F$.

If $S$ is a $G$ set and $F \subseteq E$ are fields, then a vector $v \in F[S]$ can also be viewed as lying in $E[S]$, and it is obvious that $t(v)$ does not change as we change our point of view from the field $F$ to the field $E$. Since $d(v)$ is just the dimension of the space spanned by the $G$-translates of $v$, we see by Lemma 6.2 that $d(v)$ is also invariant under this change of field.

Proof of Theorem E. The group $G$ acts transitively on the set $S$, which has prime cardinality $p$, and as we have remarked previously, it is no loss to assume that the action of $G$ on $S$ is faithful. Let $P$ be a Sylow $p$-subgroup of $G$ and note that $|P|=p$.

The $G$-module $F[S]$ can also be viewed as a $P$-module, and we observe that the $P$-submodule generated by $v$ is contained in the $G$-submodule generated by $v$. If we replace $G$ by $P$, therefore, the value of $d$ may decrease. Of course, this change has no effect on $t$, and so to prove that $t+d>p$, it is no loss to assume that 
$|G|=p$. In this case, we can assume that $S=G$ and that the action of $G$ on $S$ is regular.

If we replace the given field $F$ by the subfield generated over the rational numbers $\mathbb{Q}$ by the coefficients of $v$, this does not change either $t$ or $d$. We can thus assume that $F$ is finitely generated over $\mathbb{Q}$, and hence that $F \subseteq \mathbb{C}$. We can thus replace $F$ by $\mathbb{C}$ without changing $t$ or $d$. It therefore suffices to prove the inequality $t+d>p$ in the case where $G=S$ and $F=\mathbb{C}$. As we have seen, however, this case of Theorem E follows from Chebotarëv's theorem, and so the proof is complete.

For the following discussion, fix a prime number $p$ and let $G=S$ have order $p$. By Theorem E, we know that if the characteristic of $F$ is 0 , then the inequality $t+d>p$ holds for all nonzero vectors $v \in F[G]$. If $F$ has prime characteristic, however, this inequality can fail. The following theorem gives a necessary and sufficient condition for this failure to occur, where the condition is expressed in terms of the polynomial ring $F[X]$.

Let $z$ be a generator for $G$ and note that each vector $v \in F[G]$ can be uniquely written in the form $f(z)$, where $f \in F[X]$ and $\operatorname{deg} f<p$. The quantity $t=t(v)$ is exactly the number of nonzero coefficients in the polynomial $f$, and we write $t(f)$ to denote this number.

(6.3) Theorem. Let $G=\langle z\rangle$ be a group of prime order $p$ and suppose that $v \in F[G]$ is nonzero, where $F$ is an arbitrary field. Write $v=f(z)$, where $f \in F[X]$ and $\operatorname{deg} f<p$. Then $t(v)+d(v) \leq p$ if and only if $t(f) \leq \operatorname{deg} h$, where $h(X)=$ $\operatorname{gcd}\left(X^{p}-1, f(X)\right)$.

Before we proceed with the proof of Theorem 6.3 , we show how this result can be used to find explicit examples where $t+d \leq p$. That will enable us to find examples where Chebotarëv's theorem fails in prime characteristic.

First, factor the polynomial $X^{p}-1$ in $F[X]$ in order to determine its proper divisors. If we can find such a divisor $h(X)$ such that $t(h) \leq \operatorname{deg} h$, we are done: simply take $v=h(z)$. (Note that the condition $t(h) \leq \operatorname{deg} h$ says that the polynomial $h$ is "missing a term". In other words, the coefficient of $X^{i}$ in $h(X)$ is 0 for some exponent $i<\operatorname{deg} h$.) If no proper divisor of $X^{p}-1$ is missing a term, then consider multiples $f(X)$ of divisors $h(X)$ of $X^{p}-1$ such that $\operatorname{deg} f<p$. If it is possible to find such a multiple with $t(f) \leq \operatorname{deg} h$, then $v=f(z)$ will be the desired example.

Using a computer algebra system, it is easy to find prime numbers $p$ and finite fields $F$ such that the polynomial $X^{p}-1 \in F[X]$ has a proper divisor that is missing a term. A few such examples are given in the following table. Of course, once an example is found, the field $F$ can be replaced by any larger field, and so we list only "minimal" examples.

$$
\begin{aligned}
p=7: F & =G F(2) \\
p=11: F & =G F(3) \\
p=13: F & =G F(3), G F(4), G F(5) \\
p=17: F & =G F(2), G F(13) \\
p=19: F & =G F(4), G F(5), G F(7)
\end{aligned}
$$

We mention one further example: $p=11$ and $F=G F(5)$. In this case, no proper divisor of $X^{11}-1$ is missing a term. One such divisor, however, is $h(X)=$ 
$X^{5}+2 X^{4}+4 X^{3}+X^{2}+X+4$, and if we compute $f(X)=(X-2) h(X)$, we find that $f(X)=X^{6}+3 X^{3}+4 X^{2}+2 X+2$. Then $\operatorname{deg} f=6<p$ and $t(f)=5 \leq \operatorname{deg} h$. As we have seen, this yields an example where $t+d \leq p$.

Proof of Theorem 6.3. Suppose that $\operatorname{deg} f<p$, where $f(X) \in F[X]$, and let $h(X)=\operatorname{gcd}\left(X^{p}-1, f(X)\right)$. Assuming that $t(f) \leq \operatorname{deg} h$, we write $v=f(z)$ and we work to control $d(v)$.

Write $X^{p}-1=h(X) k(X)$ and let $s=\operatorname{deg} k$, so that $s=p-\operatorname{deg} h \geq p-$ $\operatorname{deg} f>0$. Then $X^{p}-1$ divides $f(X) k(X)$, and since $z^{p}=1$ in $G$, we see that $v k(z)=f(z) k(z)=0$. Now let $M \subseteq F[G]$ be the subspace spanned by the set $\left\{v z^{i} \mid 0 \leq i<s\right\}$ and note that $\operatorname{dim}(M) \leq s$ since the spanning set has cardinality $s$. Since $k$ has degree $s$ and $v k(z)=0$, we see that $v z^{s}$ is a linear combination of the vectors $v z^{i}$ with $0 \leq i<s$, and thus $v z^{s} \in M$. It follows that $M z \subseteq M$, and thus $M$ is a $G$-submodule of $F[G]$, and in fact, $M=\langle v\rangle$. Then $d(v)=\operatorname{dim}(M) \leq s=p-\operatorname{deg} h$ and we have $t(v)+d(v) \leq t(f)+(p-\operatorname{deg} h) \leq p$ since we are assuming that $t(f) \leq \operatorname{deg} h$.

Conversely, suppose that $0 \neq v \in F[G]$ and that $t(v)+d(v) \leq p$. Write $v=f(z)$, where $f(X) \in F[X]$ and $\operatorname{deg} f<p$. Then $t(f)=t(v)$, and we need to show that $t(f) \leq \operatorname{deg} h$, where $h(X)=\operatorname{gcd}\left(X^{p}-1, f(X)\right)$.

Choose the integer $s>0$ as large as possible such that the set $\left\{v z^{i} \mid 0 \leq i<s\right\}$ is linearly independent. Since this set is contained in $\langle v\rangle$, we clearly have $s \leq d(v)$. By the maximality of $s$, the vector $v z^{s}$ is a linear combination of vectors in our set, and so we can write $v k(z)=0$ for some polynomial $k[X] \in F[X]$ of degree $s$. Then $f(z) k(z)=0$ and it follows by the division algorithm that the polynomial $f(X) k(X)$ is a multiple of $X^{p}-1$. (This is because no polynomial of smaller degree can vanish at $z$.) Then $X^{p}-1$ divides $h(X) k(K)$, and thus

$$
t(v)+d(v) \leq p \leq \operatorname{deg} h+\operatorname{deg} k=\operatorname{deg} h+s \leq \operatorname{deg} h+d(v) .
$$

We conclude that $t(f)=t(v) \leq \operatorname{deg} h$, as desired.

As we have seen, if $F$ is a field containing a primitive $p$ th root of unity, then the conclusion of Chebotarëv's theorem over $F$ is equivalent to the assertion that $t+d>p$ for all choices of nonzero vectors $v \in F[S]$. It follows that examples where $t+d \leq p$, such as those we presented earlier, yield examples where the conclusion of Chebotarëv's theorem fails. (We need to take extensions of our minimal fields that are large enough to contain a primitive $p$ th root of unity, and this, of course, is always possible if $p$ is different from the characteristic of $F$.)

For each prime $p$, there are only finitely many characteristics where Chebotarëv can fail, and thus there are only finitely many characteristics where examples such as those discussed above can occur. To see why this is true, consider the determinants of all square submatrices of the complex matrix $\left[\zeta^{i j}\right]$, as in Theorem 6.1. These are algebraic integers, and they are nonzero by Chebotarëv's theorem, and so their norms are nonzero rational integers. It should be reasonably clear that the characteristics where the conclusion of Chebotarëv's can fail are exactly the primes that divide at least one of these integers, and clearly, there are just finitely many such primes.

We have seen several examples of primes $p$ and fields $F$ for which it is possible to find vectors $v \in F[S]$ such that $t+d \leq p$. But this cannot happen if the characteristic of $F$ is the given prime number $p$. 
(6.4) Theorem. Let $S$ be a transitive $G$-set with $|S|=p$, a prime number, and let $0 \neq v \in F[S]$, where $F$ has characteristic $p$. Then $t(v)+d(v)>p$.

Proof. As in the proof of Theorem E, we can assume that $G=\langle z\rangle$ has order $p$. By Theorem 6.3, it suffices to show that for all nonzero polynomials $f(X) \in F[X]$ with $\operatorname{deg} f<p$, we have $t(f)>\operatorname{deg} h$, where $h(X)=\operatorname{gcd}\left(X^{p}-1, f(X)\right)$. But $F$ has characteristic $p$, and thus $X^{p}-1=(X-1)^{p}$, and we see that $h(X)=(X-1)^{m}$ for some integer $m$. Our goal, therefore, is to prove that $t(f)>m$. This, however, is immediate from the following general lemma.

(6.5) Lemma. Let $0 \neq f(X) \in F[X]$, where $F$ is an arbitrary field. In the case where $F$ has prime characteristic $p$, assume in addition that $\operatorname{deg} f<p$. Suppose that $(X-1)^{m}$ divides $f(X)$, where $m \geq 0$. Then $t(f)>m$.

Proof. The result is clearly true if $m=0$, and so we assume $m>0$ and we proceed by induction on $m$. If the polynomial $f(X)$ has zero constant term, let $g(X)=f(X) / X$. Then $g(X)$ is also a polynomial divisible by $(X-1)^{m}$ and it too satisfies the degree upper bound in prime characteristic. Since $t(f)=t(g)$, we can replace $f$ by $g$. If we apply this argument repeatedly, we can assume that $f$ has a nonzero constant term.

Now $f$ is not a constant polynomial since it is divisible by $X-1$, and since in prime characteristic $p$ we have $\operatorname{deg} f<p$, it follows that the formal derivative $k(X)=f^{\prime}(X) \neq 0$. Furthermore, since $f$ has nonzero constant term, we see that $t(k)<t(f)$. Finally, we note that $k(X)$ is divisible by $(X-1)^{m-1}$, and so by the inductive hypothesis, $t(k)>m-1$. Then $t(f) \geq t(k)+1>m$, as required.

Next, we prove Theorem E again, but this time, we avoid appealing to Chebotarëv's theorem. By our remarks concerning the relationship between these two results, this will yield a new proof of Chebotarëv's theorem. We need the following lemma.

(6.6) Lemma. Let $S$ be a finite transitive right $G$-set and let $0 \neq v \in \mathbb{C}[S]$. The following then hold:

(a) There exists an algebraic number field $K$ and a nonzero vector $v_{0} \in K[S]$ such that $t\left(v_{0}\right)=t(v)$ and $d\left(v_{0}\right) \leq d(v)$.

(b) Let $p$ be any prime. Then there exists a finite field $F$ of characteristic $p$ and a nonzero vector $v_{1} \in F[S]$ such that $t\left(v_{1}\right) \leq t(v)$ and $d\left(v_{1}\right) \leq d(v)$.

To see the significance of this result, let $S$ be a transitive $G$-set with $|S|=n$ and let $\Xi(x, y)$ be a real-valued function that is monotonically increasing in each of its two real variables. Suppose we want to prove that an inequality of the form $\Xi(t, d)>n$ holds for all nonzero vectors $v \in \mathbb{C}[S]$, where $t=t(v)$ and $d=d(v)$. By Lemma 6.6(b), it suffices to prove the same inequality for all finite fields $F$ of characteristic $p$, where $p$ is some fixed prime.

We have already seen that to prove Theorem $\mathrm{E}$ we can assume that $F=\mathbb{C}$, and so by Lemma 6.6(b) it is enough to establish that the inequality $t+d>p$ always holds for finite fields of some fixed characteristic $q$, and furthermore, we get to choose $q$. But we have already done this; we can take $q=p$ by Theorem 6.4. Theorem E will thus follow once we prove Lemma 6.6, and as we have seen, Chebotarëv's result will then also follow.

We remark that we really do need Theorem 6.4, despite the fact that as we have seen, there are guaranteed to be infinitely many characteristics for which the 
inequality $t+d>p$ holds. This is because our proof that there were at most finitely many "bad" characteristics relied on Chebotarëv's theorem, which we are now trying to prove.

Proof of Lemma 6.6. Let $X$ be the $|G| \times|S|$ matrix over $\mathbb{C}$ in which the row corresponding to $g \in G$ is the vector of length $|S|$ given by the coefficients of the translate $v g$ of $v$, taken in some fixed order. Then $d=d(v)$ is the rank of this matrix, and so if $e>d$, then every $e \times e$ submatrix of $X$ will have determinant 0 .

Now let $R$ be the $\mathbb{Q}$-subalgebra of $\mathbb{C}$ generated by the $t=t(v)$ nonzero coefficients of $v$ and their reciprocals. Let $M$ be a maximal ideal of $R$ and note that the field $K=R / M$ is finitely generated as an algebra over the image of $\mathbb{Q}$ in $K$, and we identify this image with $\mathbb{Q}$. It follows by the Nullstellensatz (see Theorem 30.8 of 7]) that $K$ is a finite degree extension of $\mathbb{Q}$.

The images in $K$ of the nonzero coefficients of $v$ are invertible, and hence they are nonzero. The image of $v$, therefore, is a vector $v_{0} \in K[S]$ such that $t\left(v_{0}\right)=t$. To compute $d\left(v_{0}\right)$, we need to determine the rank of the appropriate $|G| \times|S|$ matrix $Y$ over $K$. But $X$ has entries in $R$, and $Y$ is the image of $X$ in $K$. If if $e>d$, therefore, then all $e \times e$ submatrices of $Y$ have determinant 0 . The rank of $Y$ is thus at most $d$, and we conclude that $d\left(v_{0}\right) \leq d(v)$. This establishes assertion (a).

Now $K$ is an algebraic number field, and we consider its ring $A$ of integers. Let $P \subseteq A$ be a prime ideal containing $p$ and note that $F=A / P$ is a finite field of characteristic $p$. We can replace $v_{0}$ by a nonzero scalar multiple without affecting either $d\left(v_{0}\right)$ or $t\left(v_{0}\right)$, and so by a standard fact from Dedekind domain theory, we can assume that the coefficients of $v_{0}$ all lie in $A$ but that not all of them lie in the ideal $P$. (See Lemma 29.20 of $[8$.)

Let $v_{1}$ be the image of $v_{0}$ in $F[S]$. Then $v_{1}$ is nonzero and $t\left(v_{1}\right) \leq t\left(v_{0}\right)$. Also, $d\left(v_{1}\right)$ is the rank of a certain $|G| \times|S|$ matrix $Z$ over $F$, and this matrix is the image of a matrix over $A$ whose rank (over $K$ ) is $d\left(v_{0}\right)$. Reasoning as before, we see that the determinant of every $e \times e$ submatrix of $Z$ is 0 if $e>d\left(v_{0}\right)$. It follows that $d\left(v_{1}\right) \leq d\left(v_{0}\right)$, and the proof is complete.

\section{INFINITE GROUPS}

Are there any results for infinite groups that are analogous to our inequalities? Suppose that $S$ is an infinite transitive right $G$-set and $F$ is a field. We can, of course, continue to think about the permutation module $F[S]$, which, by definition, consists entirely of vectors with finite support. But $n$ is infinite (by assumption) and if $v \in F[S]$ is nonzero, then $d$ is necessarily infinite, so what can we hope to prove? Here is an easy result that suggests that perhaps there is some theory here, although we have not pursued it beyond this very special case.

(7.1) Theorem. Let $G=\langle z\rangle$ be an infinite cyclic group acting regularly on the set $S$. Let $0 \neq v \in F[S]$, where $F$ is an arbitrary field. Then the codimension $c$ of $\langle v\rangle$ in $F[S]$ satisfies $t-1 \leq c<\infty$.

The inequality $c<\infty$ in Theorem 7.1 says that $\langle v\rangle$ is "large", and this is consistent with the inequality $d t \geq n$ that holds in the finite case. But in the finite case, $c=n-d$, and so the inequality $t-1 \leq c$ in Theorem 7.1 would correspond to an inequality of the form $t+d \leq n+1$, and this is in the opposite direction of what we might expect. (But compare this with Lemma F.) 
Proof of Theorem 7.1. If $t(v)=1$, then $\langle v\rangle=F[S]$, and so $c=0$ and we are done. We can assume, therefore, that $t(v)>1$. We prove that $c$ is finite by producing a finite subset $X \subseteq S$ such that $F[S]=F[X]+\langle v\rangle$, where, of course, the subspace $F[X] \subseteq F[S]$ is the linear span of $X$. For convenience, we think of the set $S$ as a "horizontal" linear array of points, where the generator $z$ acts by a right shift of one unit.

Since $t(v)>1$, it is possible to choose a nonempty subset $X \subseteq S$ consisting of consecutive points, such that $X$ is exactly one unit too short to contain any translate of $\operatorname{supp}(v)$. Then the subspace $F[X]+\langle v\rangle$ contains the point just to the right of the interval $X$ and also the point just to the left of this interval. It follows that $F[X] z \subseteq F[X]+\langle v\rangle$ and also $F[X] z^{-1} \subseteq F[X]+\langle v\rangle$. Then $F[X]+\langle v\rangle$ is mapped into itself by $z$ and $z^{-1}$, and hence it is a $G$ submodule of $F[S]$. We have seen, however, that $F[X]+\langle v\rangle$ contains a point of $S$, and it follows that

$$
F[X]+\langle v\rangle=F[S]
$$

as we wanted, and thus $c<\infty$.

Next, we argue that $F[X] \cap\langle v\rangle=0$ by showing that if $0 \neq w \in\langle v\rangle$, then $\operatorname{supp}(w) \nsubseteq X X$. We can write $w$ as the sum $w=\sum_{i \in I} a_{i} v z^{i}$ for some finite subset $I \subseteq \mathbb{Z}$, where the coefficients $a_{i} \in F$ are nonzero. Let $r=\min (I)$ and $s=\max (I)$ and note the $\operatorname{supp}(w)$ contains the leftmost point $x$ of $\operatorname{supp}(v) z^{r}$ and also the rightmost point $y$ of $\operatorname{supp}(v) z^{s}$. Since $r \leq s$, however, the distance between these points of $\operatorname{supp}(w)$ is at least the distance between the leftmost and rightmost points of $\operatorname{supp}(v)$, and so by the choice of $X$ it is not possible that $x$ and $y$ both lie in $X$.

It now follows that $F[S]=F[X] \dot{+}\langle v\rangle$, and so $c=\operatorname{dim}(F[X])=|X|$. By the choice of $X$, however, we see that $t \leq|X|+1$, and so $t-1 \leq c$, as wanted.

We close this section by revisiting Rudio's lemma. We mentioned in Section 2 that the strong form of Rudio's lemma does not hold in general for infinite primitive groups. We now present an example that demonstrates this.

Take $F=\mathbb{R}$, the real numbers, in Lemma 5.3, and let $A \subseteq \mathbb{R}^{\times}$be the subgroup consisting of the positive real numbers. It is clear that a subgroup of the additive group of $\mathbb{R}$ that is closed under multiplication by positive numbers will also be closed under multiplication by all real numbers, and hence there are no such subgroups other than 0 and $\mathbb{R}$ itself. It follows that the corresponding affine linear group $G$ acts primitively on $\mathbb{R}$.

Now let $X$ be the set of positive real numbers. It is easy to see that the translate of $X$ under the group element $x \mapsto a x+b$ is exactly the set $\{t \in \mathbb{R} \mid t \geq b\}$. (We are using the assumption that $a>0$ here, of course.) There is no translate of $X$, therefore, that contains 0 but not 1 , and so the strong form of Rudio's lemma fails.

\section{FURTHER REMARKS AND QUESTIONS}

We certainly have not answered, or even attempted to answer, all possible questions of the type considered in this paper. In this section, we mention some other areas for possible future research that have occurred to us.

Instead of limiting our attention to permutation modules, as we have here, we could work in a somewhat more general context. We shall say that a $G$ module $M$ over a field $F$ is monomial if it has a basis $S$ such that for each element $s \in S$ and each group element $g \in G$, the translate $s g$ is a scalar multiple of a member of $S$. (The permutation modules, therefore, are exactly those monomial modules where 
all of these scalars are 1.) In this context, we replace the assumption that $S$ is a transitive $G$-set with the requirement that the action of $G$ on the set $\{F s \mid s \in S\}$ is transitive. (We shall use somewhat nonstandard language here, and refer to these as transitive monomial modules.)

We can still define $n=|S|=\operatorname{dim}(M)$ and also $t=t(v)=|\operatorname{supp}(v)|$ and $d=d(v)=\operatorname{dim}(\langle v\rangle)$ for vectors $v \in M$, and we can hope to prove results analogous to those we have established for permutation modules. For example, the inequality $t d \geq n$ of Theorem $\mathrm{B}$ continues to hold for transitive monomial modules, and in fact, the proof of Theorem B goes through essentially unchanged. Perhaps some of our other results would also generalize to monomial modules with little or no change, but we will not pursue that here.

Another question concerns solvable primitive groups, and more generally, primitive groups having a regular normal subgroup. We have seen that if equality holds in Theorem $\mathrm{C}$ and $1<t<n-1$, then the group $G$ cannot have such a regular normal subgroup. In fact, in almost all of the primitive examples we know, if $t$ and $d$ are relatively small when compared with $n$, the group $G$ fails to have a regular normal subgroup. (The exceptions here are when $t$ is very small and when it is very nearly equal to $n$.) This suggests that substantially better inequalities might hold for primitive groups that have regular normal subgroups, and in particular, for solvable primitive groups. Perhaps in those cases some additive inequality should hold, such as that in Theorem E, which asserts that $t+d>n$ in the case where $n$ is prime.

Also, we know that equality can occur in Theorem $\mathrm{C}$ in the cases where $1<t<$ $n-1$, but only when $t=d$ and $F$ has characteristic 2 . This suggests the possibility that substantially better inequalities might hold when $|t-d|$ is large or for fields of other characteristics.

In order to find good inequalities of the type we have been discussing here, it would be useful to have more examples of extreme or nearly extreme cases. Given a nonzero submodule $M$ of the permutation module $F[S]$, we write $t(M)$ to denote $\min \{t(v) \mid 0 \neq v \in M\}$. By Lemma $\mathrm{F}$, we know that $t(M) \leq n+1-\operatorname{dim}(M)$, but what more can be said? In particular, in the case where $F$ is a finite field, it would be pleasant if we could study this question computationally, but we see no good algorithm for finding nonzero vectors $v \in M$ with small support.

We can say a bit about the effect of a change of field on the quantity $t(M)$. In fact, the following easy result has nothing to do with modules, and so we extend the definition of $t(M)$ to arbitrary vector subspaces $M$ of $F[S]$.

(8.1) Lemma. Let $S$ be a finite set and suppose that $F \subseteq E$ are fields. Let $V \subseteq F[S]$ be a nonzero $F$-subspace and write $E V$ to denote the $E$-span of $V$. Then $t(E V)=t(V)$.

Note that this result is somewhat analogous to Lemma 6.2, which was the nearly trivial observation that $\operatorname{dim}(E V)=\operatorname{dim}(V)$.

Proof of Lemma 8.1. Since $V \subseteq E V$, the inequality $t(E V) \leq t(V)$ is obvious. To prove the reverse inequality, let $\mathcal{B}$ be an $F$-basis for $V$ and let $w \in E V$ with $t(w)=t(E V)$. We can write $w=\sum_{b \in \mathcal{B}} e_{b} b$, where $e_{b} \in E$, and not all of the coefficients $e_{b}$ are 0 . If $s \in S$, then the coefficient of $s$ in $w$ is $\sum_{b \in B} e_{b} b_{s}$, where we have written $b_{s}$ to denote the coefficient of $s$ in the basis vector $b$. In particular, if $s \notin \operatorname{supp}(w)$, we have $\sum e_{b} b_{s}=0$. 
If we think of the coefficients $e_{b}$ as unknowns, we see that we have a homogeneous system of $|S|-t(w)$ linear equations in these unknowns, and the coefficients $b_{s}$ of this system lie in $F$. (Recall that $b_{s} \in F$ since $\mathcal{B} \subseteq V \subseteq F[S]$.) This system has a nontrivial solution over $E$, and it follows by elementary linear algebra that there is also a nontrivial solution over $F$. We can therefore find coefficients $c_{b} \in F$, not all 0 , such that $\sum_{b \in \mathcal{B}} c_{b} b_{s}=0$ for all points $s \in S-\operatorname{supp}(w)$. In particular, if we write $v=\sum c_{b} b$, then $0 \neq v \in V$ and $\operatorname{supp}(v) \subseteq \operatorname{supp}(w)$. Thus $t(V) \leq t(v) \leq t(w)=$ $t(E V)$, and the result follows.

(8.2) Corollary. Let $S$ be a right $G$-set and suppose that $F \subseteq E$ are fields such that each $G$-submodule $M$ of $E[S]$ is spanned over $E$ by $M \cap F[S]$. Then if $v \in E[S]$ is nonzero, there exists a nonzero vector $v_{0} \in F[S]$ such that $t\left(v_{0}\right) \leq t(v)$ and $d\left(v_{0}\right) \leq d(v)$.

Proof. Let $M=\langle v\rangle$ and write $N=M \cap F[S]$, so that $M=E N$ by hypothesis. By Lemma 8.1, we can choose a vector $w \in N$ such that $t(w)=t(N)=t(M) \leq t(v)$, where the inequality holds because $v \in M$. Also, since $w \in N$, we have $d(w) \leq$ $\operatorname{dim}_{F}(N)=\operatorname{dim}_{E}(M)=d(v)$, and the result follows.

Finally, we discuss possible improvements of Lemma 6.6(a). Recall that according to that result, if we are given a nonzero vector $v \in \mathbb{C}[S]$, where $S$ is a right $G$-set, then it is possible to find an algebraic number field $K$ and a nonzero vector $v_{0} \in K[S]$ such that $t\left(v_{0}\right) \leq t(v)$ and $d\left(v_{0}\right) \leq d(v)$. Since we can go from the complex numbers $\mathbb{C}$ down to an algebraic number field without increasing either $t$ or $d$, it seems reasonable to ask just how small a subfield we can take. If the permutation module $\mathbb{C}[S]$ happens to be multiplicity free, we can use Corollary 8.2 to get a reasonably nice answer.

(8.3) Theorem. Let $S$ be a right $G$-set and suppose that the corresponding permutation character $\pi$ of $G$ is multiplicity free. Let $F \subseteq \mathbb{C}$ be the subfield generated by the values of all of the irreducible constituents of $\pi$. If $v \in \mathbb{C}[S]$ is nonzero, then there exists a nonzero vector $v_{0} \in F[S]$ such that $t\left(v_{0}\right) \leq t(v)$ and $d\left(v_{0}\right) \leq d(v)$.

Proof. Since $\pi$ is a permutation character, it can be afforded by a rational representation, and thus for each irreducible character $\chi \in \operatorname{Irr}(G)$, the Schur index $m(\chi)$ over $\mathbb{Q}$ divides the multiplicity $[\pi, \chi]$. (See Corollary 10.2(c) of $[8$.) By assumption, each of these multiplicities is at most 1 , and thus $m(\chi)=1$ for all irreducible constituents $\chi$ of $\pi$. It follows that each such constituent is afforded by an $F$ representation, and thus there exists an $F G$-module $M$ affording the character $\pi$ and such that $M$ is the direct sum of submodules that afford distinct irreducible characters.

Since $M$ and $F[S]$ are $F[G]$-modules that afford the same character, they must be isomorphic. (For example, this follows from Problem 9.5 of $[8]$.) It follows that we can write $F[G]$ as a direct sum of submodules $M_{\chi}$ affording the distinct irreducible constituents $\chi$ of $\pi$. Since $M_{\chi}$ affords $\chi \in \operatorname{Irr}(G)$, it follows that the submodules $\mathbb{C} M_{\chi}$ of $\mathbb{C}[S]$ are distinct and pairwise nonisomorphic and that $\mathbb{C}[S]$ is the direct sum of these submodules. Every submodule of $\mathbb{C}[S]$, therefore, is the sum of some of the $\mathbb{C} M_{\chi}$. In particular, every submodule of $\mathbb{C}[S]$ is the $\mathbb{C}$-span of its intersection with $F[S]$, and we are in the situation of Corollary 8.2. The proof is now complete. 


\section{REFERENCES}

[1] W. Burnside, Theory of groups of finite order, 2nd ed., Dover Publications, New York, 1955. MR.0069818(16:1086c)

[2] P. J. Cameron, On groups of degree $n$ and $n-1$, and highly-symmetric edge colourings, J. London Math. Soc. (2) 9 (1974/75) 385-391. MR0360770 (50:13217)

[3] P. J. Cameron, Finite permutation groups and finite simple groups, Bull. London Math. Soc., 13 (1981) 1-22. MR0599634 (83m:20008)

[4] D. L. Donoho and P. B. Stark, Uncertainty principles and signal recovery, SIAM J. of Appl. Math. 49 (1989) 906-931. MR0997928 (90c:42003)

[5] R. J. Evans and I. M. Isaacs, Generalized Vandermonde determinants and roots of unity of prime order, Proc. of Amer. Math. Soc., 58 (1977) 51-54. MR0412205 (54:332)

[6] B. Huppert, Endliche Gruppen I, Springer, Berlin-New York, 1967. MR0224703 (37:302)

[7] I. M. Isaacs, Character theory of finite groups, Dover, New York, 1994. MR,1280461

[8] I. M. Isaacs, Algebra: A graduate course, Brooks/Cole, Pacific Grove, 1994. MR1276273 (95k:00003)

[9] F. Rudio, Über primitive Gruppen, Journal für reine u. angew. Math., 102 (1888) 1-8.

[10] P. Stevenhagen and H. W. Lenstra, Jr., Chebotarëv and his density theorem, Math. Intell., 18 (1996) 26-37. MR.1395088 (97e:11144)

[11] T. Tao, An uncertainty principle for cyclic groups of prime order, WorldWideWeb preprint http://xxx.arxiv.cornell.edu/pdf/math.CA/0308286

[12] A. Terras, Fourier analysis on finite groups and applications, LMS Student Texts 43, Cambridge Univ. Press, Cambridge (1999). MF1695775 (2000d:11003)

[13] H. Wielandt, Finite Permutation Groups, Academic Press, New York, 1964. MR0183775 $(32: 1252)$

Center for Communications Research, 4320 Westerra Ct., San Diego, California 92121

E-mail address: dgoldste@ccrwest.org

Department of Mathematics, University of Southern California, 1042 W. 36th Place, Los Angeles, California 90089

E-mail address: guralnic@math.usc.edu

Department of Mathematics, University of Wisconsin, 480 Lincoln Drive, Madison, WISCONSIN 53706

E-mail address: isaacs@math.wisc.edu 